1 A De Novo regulation design shows an effectiveness in altering plant secondary

\title{
2 metabolism
}

3

\section{Abstract}

5

6

$7 \quad$ Transcription factors (TFs) and cis-regulatory elements (CREs) control gene

8 transcripts involved in various biological processes. We hypothesize that TFs and

9 CREs can be effective molecular tools for De Novo regulation designs to engineer

\section{Objectives:}

We selected two Arabidopsis TF types and two tobacco CRE types to design a $D e$ Novo regulation and evaluated its effectiveness in plant engineering.

\section{Methods:}

G-box and MYB recognition elements (MREs) were identified in four Nicotiana tabacum JAZs (NtJAZs) promoters. MRE-like and G-box like elements were identified in one nicotine pathway gene promoter. TF screening led to select Arabidopsis Production of Anthocyanin Pigment 1 (PAP1/MYB) and Transparent Testa 8 (TT8/bHLH). Two NtJAZ and two nicotine pathway gene promoters were cloned from commercial Narrow Leaf Madole (NL) and KY171 (KY) tobacco cultivars. Electrophoretic mobility shift assay (EMSA), cross-linked chromatin immunoprecipitation (ChIP), and dual luciferase assays were performed to test the 
promoter binding and activation by PAP1 (P), TT8 (T), PAP1/TT8 together, and the PAP1/TT8/Transparent Testa Glabra 1 (TTG1) complex. A DNA cassette was designed and then synthesized for stacking and expressing PAP1 and TT8 together. Three years of field trials were performed by following industrial and GMO protocols. Gene expression and metabolic profiling were completed to characterize plant secondary metabolism.

\section{Results:}

PAP1, TT8, PAP1/TT8, and the PAP1/TT8/TTG1 complex bound to and activated NtJAZ promoters but did not bind to nicotine pathway gene promoters. The engineered red P+T plants significantly upregulated four NtJAZs but downregulated the tobacco alkaloid biosynthesis. Field trials showed significant reduction of five tobacco alkaloids and four carcinogenic tobacco specific nitrosamines in most or all cured leaves of engineered P+T and PAP1 genotypes.

\section{Conclusion:}

G-boxes, MREs, and two TF types are appropriate molecular tools for a De Novo regulation design to create a novel distant-pathway cross regulation for altering plant secondary metabolism.

\section{Key words: Arabidopsis; alkaloid; anthocyanin; molecular tools; tobacco;}

\section{transcription factor}




\section{Introduction}

51

Plant MYB and bHLH proteins are two large TF families with diverse regulations involved in various biological processes [1-4]. To date, TFs that regulate the anthocyanin biosynthesis have gained a comprehensive characterization in both model and non-model plants. MYB and bHLH TFs together with a WD40 protein have been demonstrated to form MBW complexes to regulate the biosynthesis of anthocyanins [5-7]. In particular, the MBW complex formed by Arabidopsis Production of Anthocyanin Pigment 1 (PAP1), Transparent Testa 8 (TT8), and Transparent Testa Glabra 1 (TTG1) has been characterized to be a main regulatory complex. PAP1, TT8, and TTG1 encode a R2R3-MYB TF (namely MYB75) [8], bHLH TF (namely bHLH42) [9], and a WD40 protein [10], respectively. Moreover, the functions of these three genes in Arabidopsis and their homologs in other plants have been intensively characterized in genetics and biochemistry [11]. PAP1 and TT8 bind to MYB recognizing elements (MREs) and G-box elements in promoters of anthocyanin pathway genes (such as DFR, ANS and 3-GT, Fig. 1 A), respectively [12-14]. The current dogma is that on the one hand, PAP1, TT8, and TTG1 form a master regulatory MYB-bHLH-WD40 (MBW) complex to only activate the anthocyanin biosynthesis in plants (Fig. 1 A) [6,15-17]; on the other hand, Arabidopsis JAZ repressors can interact with PAP1, TT8, and their MBW complex to negatively regulate the anthocyanin biosynthesis in the absence of jasmonate (JA) [18, 19]. Moreover, $P A P 1$ has been used to create red crop varieties to engineer anthocyanins, such as tobacco [20, 21], tomato [22, 23], hop [24], and canola [25]. All these studies 
reported that PAP1 alone could activate the anthocyanin biosynthesis in different tested plants. However, whether PAP1, its partner TT8, and their homologs can regulate plant secondary metabolism remains open for studies.

Tobacco is an ideal model plant to study plant secondary metabolism, given that it produces thousands of metabolites in the alkaloid, phenolic, and terpenoid families $[21,26-28]$. Nicotine is the main alkaloid of tobacco (Nicotiana tabacum) and harmfully addictive [29-36]. Other harmful tobacco alkaloids include nornicotine, anabasine, anatabine, and myosmine (Fig. 1 B). Tobacco specific nitrosamines (TSNAs) are carcinogenic compounds in tobacco smoke. Five common TSNAs are $\mathrm{N}$-nitrosonornicotine $(\mathrm{NNN})$, nicotine-derived nitrosamine ketone (NNK), N'nitrosoanatabine (NAT), N-nitrosoanabasine (NAB), and 4-(methylnitrosamino)-1-(3pyridyl)-1-butanol (NNAL) [37-39]. Given that NNN is a severe carcinogen, the Food and Drug Administration has proposed a guideline that the average level of NNN must be less than $1.0 \mathrm{ppm}$ (dry weight) in all final smokeless tobacco products [40].

To reduce these harmful compounds, numerous achievements have been accomplished in elucidating the biosynthesis of nicotine and other tobacco alkaloids [41-47]. Nicotine is biosynthesized from two distinct pathways (Fig. 1 B), the steps of which are catalyzed by enzymes encoded by 10 genes $A O, Q S, Q P T 2, O D C, A D C$, AIH, PMT, MPO, A622 and BBL, (Fig. 1 B) [42, 48, 49]. In addition, CYP82E4 and its homologs have been characterized to involve the demethylation of nicotine to form nornicotine [50-52]. The mechanisms of the anatabine and anabasine formation are unknown [42]. One hypothesis is that these two alkaloids result from the BBL 
catalysis of nicotinic acid. The other is that anabasine is derived from lysine, a different pathway (Fig. 1 B) [42]. Four main TSNAs, NNK, NNN, NAT, and NAB (Fig. 1 B), result from the nitrosation of nicotine, nornicotine, anatabine, and anabasine during the leaf curing process, respectively [42]. Meanwhile, the regulation of the nicotine pathway has gained an intensive elucidation. Myelocytomatosis oncogene (MYC2) and APETALA 2/ethylene response factor (AP2/ERF) of tobacco are two groups of transcription factors (TFs) that directly bind to promoters of nicotine pathway genes [42, 48, 53-55]. Moreover, jasmonate (JA), a plant hormone, essentially controls the nicotine biosynthesis (Fig. 1B) [56-59]. JA regulates the nicotine biosynthesis via a co-receptor complex consisting of coronatine insensitive 1 (COI1), a Skp/Cullin/F-box (SCF ${ }^{\mathrm{COI} 1}$ ) complex, and JA ZIM-domain (JAZ) repressor $[53-55,60]$. The tobacco genome has been reported to have $15 \mathrm{NtJAZs}$, in which NtJAZ1, NtJAZ3, NtJAZ7, and NtJAZ10 are associated with the regulation of the nicotine biosynthesis $[54,60,61]$. When tobacco roots lack isoleucine-JA (JA-Ile), NtJAZ1 and NtJAZ3 bind to NtMYC2 and then turn off the nicotine biosynthesis; when JA-Ile exists, it induces the interaction of $\mathrm{SCF}^{\mathrm{COI} 1}$ and JAZ to form a complex, which leads to the ubiquitination of JAZs to release NtMYC2. The freed NtMYC2 then activates the biosynthetic pathway of nicotine (Fig. 1 B) $[54,55,60]$. Compared with the appropriate characterization of NtJAZs' functions, how their transcription in tobacco is regulated remains open for investigations. To date, based on those pathway and regulatory genes, on the one hand, antisense and RNAi have been reported to down-regulate gene expression (such as $B B L, O D C, Q P T$, and nicotine demethylase gene) to reduce nicotine, nornicotine, or TSNAs in leaves $[49,55,62,63]$. On the other hand, these multiple successes revealed a challenge that the reduction of 
nicotine was traded with the increase of anatabine and other alkaloids $[64,65]$. These successful engineering data indicate that continuous efforts are necessary to diminish TSNAs, nicotine, and other alkaloids simultaneously.

Since 2000 when more than 1500 TFs were annotated in the genome of Arabidopsis thaliana in [66], 320,370 TFs had been identified from 156 sequenced plant genomes and sequences of nine additional plants by 2017 [67]. Likewise, 21,997,501 TF bind sites (motifs or regulatory elements) (TFBSs) were determined in 63 plant genomes and nearly 2,493,577 TFBSs (cis-regulatory elements) were localized in the promoters [68]. Given that the interaction of TFs and TFBSs regulates plant development, growth, responses to stresses, and metabolisms, we hypothesize that the large numbers of cis-regulatory elements and TFs are useful molecular tools for de novo designs to create novel regulations for plant engineering. For proof of concept, herein, we selected tobacco crop to test our hypothesis, given that as described above, the success might enhance diminishing harmful nicotine, other tobacco alkaloids (OTAs), and TSNAs for human health. As introduced, given that NtJAZ1 and NtJAZ3 encode two main repressors of the nicotine biosynthesis, we hypothesized that their cisregulatory elements were appropriate molecular tools to screen other plant TFs for $d e$ novo regulation designs and positive designs could be tested in the downregulation of nicotine biosynthesis in tobacco plants. Then, we analyzed cis-regulatory elements of all NtJAZ promoters and cloned MRE(s) and/or G-box(es) from four NtJAZ genes. In addition, we analyzed and identified MRE-like and G-box like elements of one nicotine pathway gene. Meanwhile, we analyzed Arabidopsis TFs that have a regulatory function to bind to these types of elements. As a result, PAP1 and TT8, two 
master regulators of Arabidopsis anthocyanin biosynthesis, were identified for this design. Mechanistic, transgenic, transcriptional, and metabolic quantification, and field farming practices showed that this design was successful to create a distant pathway-cross regulation (DPCR) crossing from the anthocyanin to nicotine pathway. The constructed DPCR showed an effectiveness in significantly reducing nicotine, four OTAs, and four TSNAs in tobacco plants, simultaneously. Moreover, the resulting data disclose new regulatory functions of both PAP1 and TT8 and new activators of four NtJAZs.

\section{Materials and methods}

\section{Tobacco varieties}

Six genotypic tobacco (Nicotiana tabacum) cultivars were used in this study. Two commercial dark tobacco varieties were Narrow Leaf Madole (NL) and KY171 for smokeless products (Andersen et al., 1990; Pearce et al., 2015). Two red transgenic genotypes were generated from NL and KY171 described below. Two others were $N$. tabacum Xanthi (an oriental tobacco variety) and a red PAP1 tobacco variety that is a novel homozygous red Xanthi variety (Fig. S22 B) created from the overexpression of the Arabidopsis PAPl (MYB75) (Xie et al., 2006). Nicotiana benthamiana was grown for dual-luciferase assay. Growth conditions in the greenhouse and phytotron are listed in supporting materials. 
Cloning of NtJAZ1, NTJAZ3, PMT2, and ODC2 promoters from Narrow Leaf Madole and KY171 cultivars

The promoter sequences of tobacco NtJAZ1, NtJAZ3, NtJAZ7, NtJAZ10, PMT2, and $O D C 2$ were identified from the genomic sequences of the NT90 cultivar curated at NCBI (https://www.ncbi.nlm.nih.gov/). The sequences identified were 1000 bp or $1500 \mathrm{bp}$. Based on these sequences, primers (Table S1) were designed for PCR to amplify NtJAZ1, NtJAZ3, PMT2, and $O D C 2$ promoters from the genomic DNA of wild type NL and KY171 tobacco plants, and then the amplified genomic DNA fragments were cloned to pEASY-T1 for sequencing. Briefly, the DNA fragments from PCR were purified using a gel-extraction Kit (Thermo Fisher, Waltham, MA) by following the manufacturer's instruction and then ligated to the pEASY-T1 plasmid using a T4-ligase system ( $10 \mu$ l reaction system: $1 \mu 1$ T4 buffer, $1 \mu 1$ T4-ligase, $1.0 \mu 1$ pEASY-T1 linear plasmid and $7.0 \mu \mathrm{l}$ purified PCR product). The resulting recombinant plasmid was transformed into the competent $E$. coli Top10 cells, which were screened on agar-solidified LB medium supplemented with $50 \mathrm{mg} / \mathrm{l}$ ampicillin. Positive colonies were selected for sequencing at Eton Bio (Durham, NC, USA). All promoter sequences obtained were 1000 or 1500 bp (Figs. S1, S2, and S4). Analysis of promoter sequences and identification of regulatory elements such as AC- and AClike elements (Figs. S1, S2, and S4) were completed with the PLACE (http://www.dna.affrc.go.jp/PLACE/) and PlantPan (http://plantpan.itps.ncku.edu.tw/) tools. The NtJAZ1, NtJAZ3, PMT2, and ODC2 promoters were used for electrophoretic mobility shift assay, dual-luciferase, and ChIP assays described below. 
192

193

Electrophoretic mobility shift assay of PAP1 and TT8 binding to promoters

To perform electrophoretic mobility shift assay (EMSA), we designed gene-specific primers (Supporting Table S1) for PCR to amplify the binding domains of both PAPI and TT8. The amplified PAPl binding domain from the N-terminus was $534 \mathrm{bp}$, which included the $R 2 R 3 M Y B$ domain sequences for Gate-way cloning. The amplified TT8 binding domain franking the $b H L H$ region was $252 \mathrm{bp}$, in which both BamHI and SaclI restriction sites were added to two ends for further cloning. After the confirmation of sequence accuracy, three expression vector systems, pRSF-Dute, pet-Dute, and pDest-His-MBP were comparatively tested to induce soluble recombinant proteins. We selected the pDest-His-MBP vector, a Gateway cloning system, for the $R 2 R 3$ domain of $P A P 1$. We found that the pRSF-Dute vector with a His-tag in the N-terminus was appropriate for expressing the bHLH domain of TT8 after several vectors were tested. As reported previously[69], detailed steps are listed in Method S1.

Dual luciferase assays

Four reporter and four effector vectors were developed to perform dual luciferase assays that were completed to analyze the regulatory activity of PAP1 alone, TT8 alone, PAP1 and TT8 together, and PAP1/TT8/TTG1 (WD40) together in activating NtJAZ1, NtJAZ3, PMT2, and ODC2 promoters. First, the pGreenII-0800 vector was used to develop four reporter vectors. Next, four effector vectors were developed from the PK2GW7 vector [20], to which the ORFs of GFP, PAP1, TT8, and TTG1 were 
cloned. Each gene was driven by $35 \mathrm{~S}$ promoter. Then, four reporter and four effector vectors were introduced into Agrobacterium tumefaciens strain GV3101, respectively. Last, the activated effector and reporter Agrobacterium GV3101 cells were used to inoculate leaves of $N$. benthamiana for transient expression assay. Detailed steps are described in Method S2.

Cross-linked chromatin immunoprecipitation (ChIP) assays

A gateway cloning was used to develop two plasmids, in which GFP was fused to the 3'-end of PAPI and TT8 ORFs without their stop codon for genetic transformation. A pair of primers (Supporting Table S1) was designed to amplify the PAPl ORF without its stop codon. The resulting PCR product was purified as described above and then cloned into the $\mathrm{pDonr} 221$ vector via a $\mathrm{BP}$ reaction. The PAP1 fragment was further cloned to the upstream of GFP in the destination vector pGWB5 via a LR reaction. This cloning step generated a recombinant vector pGWB5-PAP1-GFP, in which PAP1-GFP was driven by 35 S promoter. The pGWB5-PAP1-GFP vector was introduced into the competent $E$. coli Top10 cells. Positive colonies were selected on LB medium containing $50 \mathrm{mg} / \mathrm{l} \mathrm{kanamycin.} \mathrm{One} \mathrm{colony} \mathrm{was} \mathrm{used} \mathrm{for} \mathrm{suspension}$ culture on the same shaker as described above. Cultured $E$. coli cells were harvested to isolate the pGWB5-PAP1-GFP binary plasmid, which was further introduced into competent Agrobacterium GV301 cells. To develop a binary vector expressing TT8, a pair of primers (Supporting Table S1) was designed for PCR. The reverse primer was designed to include a HA-tag sequence. The resulting PCR product was cloned to the plasmid PK2GW7 as described above. Next, the resulting binary vector PK2GW7TT8-HA-tag was introduced into competent Agrobacterium GV301 cells. 
Agrobacterium GV301 cells harboring PAP-GFP or TT8-HA-tag cDNA were activated and then injected to leaves of 5-week old wild type NL and KY171 seedlings that were grown in the phytotron. After the injection, plants were allowed to grow 72 hrs. The infected leaves were then harvested to liquid nitrogen to isolate nuclei and ChIP experiments. Detailed steps are listed in Method S3.

Synthesis of T-DNA, development of binary constructs, and genetic transformation of two dark tobacco varieties

PAP1 and TT8 of A. thaliana encode a R2R3-MYB and bHLH TF, respectively. A DNA cassette was designed and then synthesized to stack PAP1 and TT8 for their coupled overexpression. From 5' to 3' end, the cassette was composed of attL1, the PAP1 cDNA (NP_176057), NOS (terminator), 35S promoter, the TT8 cDNA (CAC14865), and attL2 (Fig. S7 A). The synthesized sequence was introduced to the entry vector pUC57 and then cloned to the destination binary vector pK2GW7 by attL $\times$ attR combination (LR) reaction with LR clonase. The resulting binary vector, namely PAP1-TT8-pK2GW7 (Fig. S7 B), was further introduced into the competent cells of Agrobacterium tumefaciens strain GV3101. A positive colony was selected for genetic transformation of tobacco. In addition, the pK2GW7 vector was used for genetic transformation as control. These constructs were transformed into two globally commercial dark tobacco varieties, NL and KY171, respectively. Detailed steps (Fig. S7 C) for transformation and selection of T0 transgenic plants followed our protocols reported previously [20]. Transgenic plants from NL and KY171 were labeled as $\mathrm{P}+\mathrm{T}-\mathrm{NL}$ and $\mathrm{P}+\mathrm{T}-\mathrm{KY}$, respectively. More than $20 \mathrm{~T} 0$ transgenic plants 
were obtained for each variety and grown in the greenhouse to select T0 seeds, 12 of

which were further selected on medium supplemented with antibiotics to screen antibiotic resistant $\mathrm{T} 1$ progeny. A 3:1 ratio of seed germination to seed death indicated one single copy of T-DNA that was in the genomic DNA of T0 plants. This ratio was observed in the $\mathrm{T} 1$ progeny of $\mathrm{T} 0$ plants. In this study, we focused on lines with one single copy of transgenes. A large number of seeds were obtained from the $\mathrm{T} 1$ progeny of each line. Seeds from these types of T1 progeny were screened on an agar-solidified MS medium supplemented with $50 \mathrm{mg} / \mathrm{l} \mathrm{kanamycin}$. If all seeds from a T1 plant could germinate $\mathrm{T} 2$ seedlings, it indicated that this $\mathrm{T} 1$ plant was a homozygous line. Accordingly, red homozygous T2 plants (Fig. S7 M) were obtained and then used for growth in the greenhouse and field trial. In addition, PCR and qRTPCR were performed to demonstrate the presence of transgene in the genome of T0 and $\mathrm{T} 2$ transgenic plants and the expression of both PAP1 and TT8 in both $\mathrm{P}+\mathrm{T}-\mathrm{NL}$ 278 and $\mathrm{P}+\mathrm{T}-\mathrm{KY}$ plants.

DNA and RNA extractions

Genomic DNA was extracted using a DNeasy Plant Mini Kit (QIAGEN, Hilden,

283 Germany). RNA was extracted using Trizol extraction reagents. Fresh plant samples were ground into fine powder in liquid nitrogen. Extraction steps were followed the manufacturers' protocols. Detailed steps are listed in Method S4. 
290 All real-time quantitative RT-PCR (qRT-PCR) analyses were performed for wild-

291 type, vector control transgenic, and red transgenic plants. SYBR-Green PCR Master-

292 mix (Bio-Rad, Hercules, CA) was used for qRT-PCR experiments on a Step-One

293 Real-time PCR System (Thermo Fisher, Waltham, MA). Detailed steps are listed in

294

295 Method S5.

\section{Assays of anthocyanin and proanthocyanidin in seedlings}

Fresh leaves and roots (100 mg) of wild type, P+T-NL1, P+T-KY1, and vector control seedlings grown hydroponically (Supporting Figure S9) were used to extract anthocyanins and proanthocyanidins (PAs). Methods were followed our anthocyanin [20] and proanthocyanidin assay protocols [70]. Detailed steps are listed in Method S6.

Field trial of $P+T-N L 1$ and $P+T-K Y 1$ T2 progeny and air curing

\section{Homozygous T2 progeny of P+T-NL1 and P+T-KY1 were planted at the research} station in Oxford, North Carolina in 2015. In addition, NL and KY171 plants were planted as controls. Prior to planting transgenic plants in the field, we applied for GMO permits from USDA-APHIS for this field trial. North Carolina State University was licensed to grow transgenic tobacco plants in the field. Every single step strictly followed the protocol requested in the license. Field planting also followed the protocol of commercial tobacco production in North Carolina. These steps included 
seedling growth in a contained greenhouse, selection and design of field, planting, phenotypic observation, field management, topping, harvest of leaves, air curing, and cleanup of plant residues from the field (Table S2 and Supporting Figures S15-S19). Detailed steps are listed in Method S7 (Table S2, Figs S15-S19).

Field trial of red PAP1 tobacco plants and flue-curing

PAP1 tobacco is the progeny of a homologous red PAP1 Xanthi plant (\#292) that is generated by the overexpression of PAP1 [21]. The field trials for PAP1 tobacco were conducted in 2011 and 2012. Field design, seed germination, plantation, field management, and plant growth management were the same as described above for P+T-NL1 and P+T-KY1 genotypes. The main differences were that leaves were harvested at different dates and flue-cured in barns. Detailed steps are listed in Method S8 (Fig. S22 A-E).

Determination of TSNAs in cured leaves of field-grown P+T-NL1 and P+T-KY1 tobacco plants with high performance liquid chromatography coupled with triple quadrupole tandem mass spectrometer

Accurate quantification of tobacco specific nitrosamines (TSNA) was completed with high performance liquid chromatography coupled with triple quadrupole tandem mass spectrometer (HPLC-QQQ-MS/MS). This analysis was performed using industrial protocols on an Agilent 1200 series HPLC System coupled with AB Sciex Triple quadrupole mass spectrometer (API 5000 with Electrospray) at RJ Reynolds. Four 
TSNAs, N-nitrosonornicotine (NNN), N-nitrosoanatabine (NAT), N-nitrosoanabasine (NAB), and nicotine-derived nitrosamine ketone (4(methylnitrosamino)-1-(3-pyridyl)1-butanone, NNK) in air-cured leaves, were quantified. One hundred milligrams of dry leaf powder were extracted in an aqueous ammonium acetate solution $(100 \mathrm{mM}$ aqueous ammonium acetate containing four deuterium analogs for TSNA) and filtered through disposable PVDF syringe filters into autosampler vials. The extract was separated in a Phenomenex Gemini C18 column with $3.0 \mu \mathrm{m}$ particle $2.0 \times 150 \mathrm{~mm}$. The injection volume was 2.0-5.0 $\mu$ l. TSNAs were detected by multiple reaction monitoring (MRM) of the precursor ion to a product ion specific for each compound. Quantification was achieved using an internal standard calibration comprised of ten points. A separate internal standard was used for each analyte by using a mixture of four stable isotope-labeled analytes. Results are reported in units of ng/g (ppb). Data were determined to be acceptable if the correlation coefficient for the calibration curve was greater than 0.99 , standards were within $85 \%$ to $115 \%$ of expected concentration values, check solution values were within $75 \%$ to $125 \%$ of target, peak shape and resolution were acceptable (based on historical data), and values for the QC samples were within established control limits. The instrument parameters for MS, HPLC gradient, the quadrupole mass spectrometer parameters, reagents and standards are listed in in Supporting Tables S3-S6.

\section{Determination of nicotine and other tobacco alkaloids in cured leaves of field grown} $P+T-N L 1$ and $P+T-K Y 1$ tobacco plants by $G C$

Analysis of gas chromatograph (GC) equipped with a flame ionization detector (FID) was performed using a standard industry method to quantify nicotine and other 
tobacco alkaloids at RJ Reynolds. In this study, analysis of nicotine and other tobacco alkaloids was performed on an Agilent 6890 gas chromatograph (GC) equipped with a flame ionization detector (FID) and an Agilent 7683 automatic sampler. One hundred milligrams of air-cured dry leaf powder were alkalinized in $2 \mathrm{mM}$ sodium hydroxide $(\mathrm{NaOH})$. This solution was extracted with methyl-tertbutyl ether (MTBE) spiked with an internal standard using a wrist-action shaker. The mixture was allowed to separate to two layers. The resulting MTBE layer was transferred to an autosampler vial for analysis of nicotine, anabasine, nornicotine, and myosmine with GC-FID.

Quantification was achieved using an internal standard calibration comprised of six points. Data were determined to be acceptable if the correlation coefficient of the calibration curve was greater than 0.998 , the response factors $(\mathrm{RF})$ were consistent, the percent relative standard deviation (\%RSD) for RF was equal or less than 5\%, the check solution values were within $10 \%$ of target, values for the quality control (QC) samples were within established control limits, and chromatograms had appropriate identification of peaks. The instrument parameters, the oven program, and reagents and conditions used in alkaloid analysis are listed in Supporting Table S7-S9. The results were reported as percentiles (\%, g/g, dry weight).

\section{Determination of TSNAs and tobacco alkaloids in flue-cured leaves of PAP1 plants} with HPLC-ESI-MS

\section{HPLC-ESI-MS analysis was performed on a 2010EV LC/UV/ESI/MS instrument} (Shimadzu, Japan). One gram of powdered samples was extracted in $20 \mathrm{ml}$ of methanol in a flask placed on a shaker for one hour at room temperature. The samples were centrifuged twice at $4000 \mathrm{rpm}$ and aliquots of the supernatant were transferred to 
387

a $2.0 \mathrm{ml}$ glass vial. The samples were separated on an Eclipse XDB-C18 analytical column $(250 \mathrm{~mm} \times 4.6 \mathrm{~mm}, 5 \mu \mathrm{m}$, Agilent, Santa Clara, CA, USA). The mobile phase solvents were composed of $1 \%$ acetic acid in water (solvent A; HPLC-grade acetic acid and LC-MS grade water) and 100\% LC-MS grade acetonitrile (solvent B). To separate metabolites, the following gradient solvent system was used with ratios of solvent A to B: 90:10 (0-5 min), 90:10-88:12 (5-10 min), 88:12-80:20 (10-20 min), 80:20-75:25 (20-30 min), 75:25-65:35 (30-35 min), 65:35-60:40 (35-40 min), 60:40-50:50 (40-55 $\mathrm{min})$, and 50:50-10:90 (55-60 min). The column was then washed for 10 min with $10 \%$ solvent $\mathrm{B}$. The flow rate and the injection volume were $0.4 \mathrm{ml} / \mathrm{min}$ and $20 \mu \mathrm{l}$, respectively. The total ion chromatograms of positive electrospray ionization were recorded from 0 to $60 \mathrm{~min}$ by mass spectrum detector and mass spectra were scanned and stored from $\mathrm{m} / \mathrm{z}$ of $120-1,600$ at a speed of 1,000 amu/s. Standards of NAB, NAT, NNAL, NNK, and NNN (Sigma, St-Louis, MO) were used to establish standard curves with a coefficient value at least $98 \%$. The peak values were used to estimate the levels of nicotine, nornicotine, anabasine, and anatabine.

Determination of nicotine and nornicotine in leaves and roots of seedlings with HPLC-qTOF-MS

Seedlings of P+T-NL1, P+T-KY1, vector control, and wild type plants were hydroponically grown in the phytotron. Leaves and roots of 45-day old seedlings (Supporting Figure S9) were used to analyze nicotine and nornicotine in both leaves and roots. Fresh tissues were ground into fine powder in liquid nitrogen and dried in a 
411

$45^{\circ} \mathrm{C}$ oven. One hundred $\mathrm{mg}$ of powdered samples was extracted with $2.0 \mathrm{ml}$ methanol. Other steps were as described above. The analysis of nicotine and nornicotine was performed with HPLC-qTOF-MS on Agilent 6520 time-of-flight LCMS/MS instrument (Agilent Technologies, Santa Clara, CA, USA). Mobile solvents and column used for separation of metabolites were solvent A and B described above. The gradient solvent system was composed of ratios of solvent A to B: 95:5 (0-5 $\min$ ), $95: 5$ to $90: 10(5-10 \mathrm{~min}), 90: 10$ to $85: 15$ (10-15 $\mathrm{min}), 85: 15$ to $45: 55$ (15-20 $\min$ ), $45: 55$ to $25: 75$ (20-25 $\mathrm{min}), 25: 75$ to $95: 5$ (25-26 min). After the last gradient step, the column was equilibrated and washed for 10 min with solvents A: B (95:5). The flow rate was $0.4 \mathrm{ml} / \mathrm{min}$. The injection volume of samples was $5.0 \mu \mathrm{l}$. The dry gas flow and the nebulizer pressure were set at $121 / \mathrm{min}$ and at $50 \mathrm{psi}$, respectively. Metabolites were ionized with the negative mode. The mass spectra were scanned from 100 to $3000 \mathrm{~m} / \mathrm{z}$. The acquisition rate was three spectra per second. Other MS conditions included fragmentor: $150 \mathrm{~V}$, skimmer: $65 \mathrm{~V}$, OCT $1 \mathrm{RF}$ Vpp: $750 \mathrm{~V}$, and collision energy: 30 psi. Standard curves were developed for both nicotine and nornicotine. The results were reported as $\mathrm{mg} / \mathrm{g}$, dry weight.

\section{Statistical analysis}

Metabolite values for each group of samples such as B1 were averaged from samples of three plots. One-way ANOVA was used to evaluate the statistical significance of the contents of nicotine, nornicotine, anabasine, NNN, NNK, NAT, and NAB between red and wild type leaves. Standard deviation was used to show the variation of metabolite contents. For gene expression, protein analysis, nicotine and nornicotine 
435

436

437

contents in seedlings, all values are averaged from four or five biological replicates.

Student t-test was performed to evaluate statistical significance. P-values less than $0.05,0.01$, and 0.001 mean significant, very significant, and extremely significant.

\section{Results}

MRE and G-box elements identified in the promoters of NtJAZ1, 3, 7, and 10

NtJAZ1 and NtJAZ3 are two main repressors of nicotine biosynthesis [54, 55, 60]. In recent, totally $15 \mathrm{NtJAZs}$ were mined from the tobacco genomes, showing that NtJAZ7, and NtJAZ10 were also associated with the regulation of nicotine biosynthesis [61]. We identified the promoter sequences of $13 \mathrm{NtJAZs}$ from the genome of NC90 tobacco curated at NCBI and named these as NtJAZ1pro through NtJAZ13pro. One or $1.5 \mathrm{~kb}$ nucleotides in the proximal upstream from the start codon (ATG) were obtained for sequence characterization. Sequence analysis revealed that NtJAZ1pro, NtJAZ3pro, and NtJAZ7pro had both MYB recognizing element (MRE) and G-box elements, while NtJAZ10pro only had G-box elements (Figs S1-S2). Next, we cloned the promoter sequences of NtJAZIpro and NtJAZ3pro from two commercial smokeless dark tobacco cultivars, Narrow Leaf Madole (NL) and KY171 (KY). Their sequences were identical to those of NtJAZ1pro and NtJAZ3pro from TN90 (Fig. S1), therefore, we used the same names in this report. Sequence analysis further substantiated the MRE and G-box elements in NtJAZ1pro and NtJAZ3pro. NtJAZ1pro has two MRE and two G-box elements (Fig. 2 A and Fig. S1). The two MRE elements are MRE1: $\underline{\text { ACCCACC }}$ at position -180 and MRE2: $\underline{\mathrm{ACCCCAC}}$ at 
459

460

461

462

463

464

465

466

467

468

469

470

471

472

473

474

475

476

477

478

479

480

481

482

position -172. The two G-boxes are $\underline{\text { CACGTG }}$ at positions -232 and AACGTG at 395. NtJAZ3pro has one MRE3 element: AACTACC at position -169 and one G-box:

CACGTG at position -960 (Fig. 2 A and Fig. S1).

EMSA shows the binding of PAP1 and TT8 to the NtJAZ1 and NtJAZ3 promoters in vitro

Electrophoretic mobility shift assay (EMSA) was carried out to test whether PAP1 and TT8 could bind to the MRE and G-box elements of NtJAZ1 pro and NtJAZ3 $3_{\text {pro }}$ in vitro. The R2R3-MYB binding domain (amino acids 1-178) of Arabidopsis PAP1 was cloned to the pDEST-HisMBP vector, in which it was fused with the His-MBP-Tag at the N-terminus (Fig. S3 A). The bHLH binding domain (amino acid 359-443) of TT8 was cloned to the PRSF-Dute vector, in which it was fused with the His-Tag at the Nterminus (Fig. S3 B). Two constructs were introduced to $E$. coli to induce recombinant proteins, which were further purified with a His-tag purification system (Fig. S3 B). Biotin probes were prepared for MRE1 (ACCCACC), MRE2 (ACCCCAC), MRE3 (AACTACC), and G-box (CACGTG). Both competitive and non-competitive probes were used as controls for EMSA. The resulting data showed that the recombinant R2R3 binding domain directly bound to three MRE element probes and the recombinant bHLH binding domain directly bound to the G-box elements (Fig. 2 B). The binding activity of both recombinant R2R3 and bHLH binding domains was competed by competitive probes but not competed by noncompetitive probes (Fig. 2 B). These data demonstrate that both PAP1 and TT8 bind to the NtJAZ1 pro and NtJAZ3 pro. 
ChIP-Q-PCR analysis was performed to examine whether PAP1 and TT8 could bind to NtJAZ1pro and NtJAZ3pro in vivo. In ChIP experiments, PAP1 was fused at the 5'-

489 end of GFP in the binary vector pGWB5 to obtain pGWB5-PAP1-GFP, which was introduced into leaves of wild type NL and KY171 seedlings for transient expression via injection of Agrobacterium. TT8 was fused at the 5'-end of an HA-tag sequence in the binary vector PK2GW7 to obtain PK2GW7-TT8-HA-tag, which was also introduced into leaves of wild type NL and KY171 seedlings for transient expression by injection of Agrobacterium. The IP experiments of PAP1 and TT8 were performed by using GFP and HA monoclonal antibodies. The ChIP PCR results showed that the amplified NtJAZ1 and NtJAZ3 promoter fragments were increased 5.0-40.4 times by PAP1 and 6.1.8-37.5 times by TT8 (Fig. 2 C).

Dual-luciferase assay demonstrates the activation of NtJAZ1 and NtJAZ3 promoters by PAP1, TT8, PAP1/TT8, and PAP1/TT8/TTG1

Dual-luciferase assay was completed to demonstrate whether PAP1 alone, TT8 alone, PAP1 and TT8 together, and PAP1/TT8/TTG1 (WD40) together could activate the NtJAZ1 and NtJAZ3 promoters in tobacco. PAP1, TT8, and TTG1 were used as effectors. The ORF sequences of $P A P 1, T T 8, T T G 1$, and GFP were respectively cloned into the PCB2004 plasmid to generate four constructs, in which each gene was 
507

508

driven by a 35S-promoter (Fig. 2 D). The NtJAZ1pro and NtJAZ3pro were cloned into the plasmid pGreenII-0800 to drive the luciferase gene to obtain NtJAZ1pro-luciferase and NtJAZ3pro-luciferase reporters (Fig. 2 D). Effectors and reporters were incubated for dual-luciferase assays. Promoters alone and a binary vector were used as controls. The resulting data showed that compared with controls, PAP1 and TT8 alone enhanced the activity of NtJAZ1pro 3.8 and 7.2 times, respectively, and increased the activity of NtJAZ3pro 1.9 (slightly) and 2.1 (significantly) times, respectively (Fig. 2 E). PAP1 and TT8 together enhanced the activity of NtJAZ1pro and NtJAZ3pro approximately 4.0 and 6.3 times (Fig. 2 E). Furthermore, PAP1, TT8, and TTG1 together increased 12.7 and 15.6 times of the activity of both NtJAZ1pro and NtJAZ3pro, much higher than PAP1 alone, TT8 alone, and PAP1 and TT8 together (Fig. 2 E). These results demonstrate that PAP1 and TT8 alone, PAP1 and TT8 together, PAP1-TT8-TTG1 complex transcriptionally activate both NtJAZ1 and NtJAZ3.

PAP1, TT8, PAP1/TT8, and PAP1/TT8/TTG1 do not activate promoters of ODC2 and PMT of tobacco

$N t O D C 2$ and NtPMT2 are two key upstream pathway genes of the nicotine biosynthesis. We obtained $1.0 \mathrm{~kb}$ long sequences for these two genes' promoters from the tobacco genome database curated at NCBI. The promoter sequence of NtPMT2 has an MRE-like element ( $\underline{\text { ACAACC }}$ at position -530) and a G-box like ( $\underline{\text { ACGTT }}$ at position -186) element, while the sequences of the $N t O D C 2$ promoter does not have MRE and G-box elements (Fig. S4). 
532 EMSA experiments were performed to test whether PAP1 and TT8 could bind to

533 MRE-like and G-box-like elements. The EMSA results indicated that the TT8 binding

534 domain could bind to the G-box-like (CACGTT) element. However, when a

535 competitive probe was added, the binding signal was lost (Fig. S5 A). Furthermore,

536 compared with the positive control G-box elements from NtJAZ1 and NtJAZ3

537 promoters, the resulting data showed that the signal from G-box like element was

538 much weaker (Fig. S5 A). This result indicates that TT8 can weakly bind to the

539 promoter of NtPMT2. When the MRE-like element probe was prepared for EMSA,

540 the resulting data showed that PAP1 could not bind to the MRE-like probe

541 (AACAACC), while PAP1 strongly bound to the positive control MRE1 and MRE3

542 probes of NtJAZ1 and NtJAZ3 promoters (Fig. S5 B).

543

544 Dual-luciferase assay and ChIP-Q-PCR were performed to test whether PAP1 and

545 TT8 could activate the $O D C 2$ and $P M T 2$ promoters. The dual-luciferase assay data

546 did not show that PAP1 alone, TT8 alone, PAP1/TT8 together, and the

547 PAP1/TT8/TTG1 complex could activate the activity of the two promoters (Fig. S6

548 A). Meanwhile, the ChIP-Q-PCR data showed that PAP1 and TT8 could not enrich

549 binding elements of the two promoters in vivo (Fig. S6 B). These data indicate that

550 PAP1, TT8, PAP1/TT8, and PAP1/TT8/TTG1 cannot activate the promoters of these

551 two key pathway genes.

552

553

Engineering of red genotypic $P+T-N L$ and $P+T-K Y$ tobacco plants

554 
An expression cassette was synthesized to stack the ORF sequences of PAPl (P) and TT8 (T) and then used to generate $20 \mathrm{~T} 0$ transgenic plants from each of Narrow Leaf Madole (NL) and KY171 (KY), two major commercial varieties (Fig. S7 A, B, C, E, and G-H). In addition, vector control transgenic plants were generated (Fig. S7 D and F). The first 12 red T0 transgenic plants from the two cultivars were tagged as $\mathrm{P}+\mathrm{T}-$ NL1 to 12 and P+T-KY1 to 12 , respectively. The first 12 T0 vector control transgenic plants from the two cultivars were tagged as NL1 to 12 and KY1 to 12, respectively. These T0 plants were selected to obtain seeds to screen T1 and homozygous T2 progeny (Fig. S7 I-M), during which the copy number of the transgenes was determined. Five P+T-NL lines (1-5) and five P+T-KY lines (1-5) were confirmed to contain one single copy of the stacked two transgenes. Quantitative RT-PCR showed that homozygous T2 progeny highly expressed PAP1 and TT8 (Fig. S8). Furthermore, qRT-PCR was performed to understand the expression of $C H S, C H I, F 3 H, F 3$ ' $H$, $D F R$, and ANS involved in the biosynthesis of both anthocyanin and proanthocyanidin (PA) and the expression of 3-GT involved in the glycosylation of anthocyanidins (Fig. 1 A) in T2 seedlings (Fig. S9). The resulting data showed that the expression levels of these seven genes were up-regulated 2-1000 times (Fig. S10 AB) in pink roots and red leaves of the P+T-NL and P+T-KY genotypes. Further UV spectrometry-based measurements showed that the roots and leaves of analyzed P+TNL1 and P+T-KY1 genotypes produced anthocyanins, while those of control plants did not (Fig. S11). It was noticeable that the levels of anthocyanins were significantly higher in leaves than in roots of both P+T-NL1 and P+T-KY1 plants (Fig. S11). To understand this differentiation, the expression of $A N R$ specifically involved in the colorless PA formation [71] was analyzed. The resulting data showed that the expression of ANR 
was significantly activated in roots but not in leaves of the two red genotypes (Fig. S10). To substantiate this, root extracts of both $\mathrm{P}+\mathrm{T}-\mathrm{NL} 1$ and $\mathrm{P}+\mathrm{T}-\mathrm{KY} 1$ seedlings were treated with DMACA. A bluish color was immediately developed from the reaction (Fig. S12 A-B), showing the presence of PAs. However, this bluish color was not developed from the extracts of both P+T-NL1 and P+T-KY1 leaves and wild type roots and leaves, indicating the lack of PAs. Further measurement of DMACA-treated samples at $640 \mathrm{~nm}$ showed that the ABS values were 5-6 times higher in the root extracts than in the leaf extracts of P+T-NL1 and P+T-KY1 and in the leaf and root extracts of wild type (Fig. S12 C-D). These data showed that the less red pigmentation in roots resulted from the conversion of red anthocyanidins to colorless PAs by ANR. In addition, the less red pigmentation in roots might result from the lack of light in the hydroponic condition and secretion of anthocyanins.

Upregulation of NtJAZ1, 3, 7, and 10 and downregulation of the nicotine biosynthesis in red $P+T-N L$ and $P+T-K Y$ plants

Both transcriptional and metabolic profiling were performed to characterize the biosynthesis of nicotine in homozygous T2 seedlings (Fig. S9) of different genotypes selected, including P+T-NL1, P+T-NL2, P+T-NL3, P+T-KY1, P+T- KY 2, and P+TKY3. Quantitative RT-PCR was performed to characterize the transcriptional profiles of 16 genes (Fig. $1 \mathrm{~B}$ ) involved in the nicotine biosynthesis. The expression levels of NtJAZ1, NtJAZ3, NtJAZ7, and NtJAZ10 were significantly increased in roots of both P+T-NL and P+T-KY genotypes (Fig. 3 A-B and K-L, Fig. S13). The expression levels of PMT1, PMT2, MPO, QPT2, A622, BBL and ERF189 were significantly 
603

604

605

606

607

608

609

610

611

612

613

614

615

616

617

618

619

620

621

622

623

624

625

626

reduced in roots of both P+T-NL (Fig. 3 D-J) and P+T-KY plants (Fig. 3 N-T). The expression levels of $O C D 2$ was not altered in the P+T-NL1, 2, and 3 lines (Fig. 3 C) but significantly decreased in the P+T-KY1, 2 and 3 lines (Fig. $3 \mathrm{M}$ ), indicating the different responses of this gene expression in two commercial varieties to transgenes. The expression levels of $M Y C 1 a, M Y C 1 b, M Y C 2 a$, and $M Y C 2 b$ were not altered in roots of both $\mathrm{P}+\mathrm{T}-\mathrm{NL}$ and $\mathrm{P}+\mathrm{T}-\mathrm{KY}$ plants (Fig. S14). HPLC-MS analysis showed that the contents of nicotine, nornicotine, and the total amount of the two compounds in roots and leaves of $\mathrm{P}+\mathrm{K}-\mathrm{NL} 1, \mathrm{P}+\mathrm{K}-\mathrm{NL} 2, \mathrm{P}+\mathrm{K}-\mathrm{NL} 3, \mathrm{P}+\mathrm{K}-\mathrm{KY} 1, \mathrm{P}+\mathrm{K}-\mathrm{KY} 2$, and $\mathrm{P}+\mathrm{K}-$ KY3 plants were significantly reduced $1.5-3.4,1.3-2.5$, and 1.9-3.3 fold, respectively (Fig. 4).

Field trials and reduction of nicotine, nornicotine, anatabine, anabasine, myosmine, and four TSNAs in red $P+T-N L 1$ and $P+T-K Y 1$ tobacco plants

Field trials that follow the commercial production practices of tobacco are essential to examine the effects of new varieties or genes on the contents of nicotine, other tobacco alkaloids, and TSNAs. In addition, field farming practices were necessary to test the agricultural significance of this de novo regulation design. The field practice exactly followed the protocols of both industry tobacco and GMO tobacco growth. We planted T2 homozygous $\mathrm{P}+\mathrm{T}-\mathrm{NL} 1$ and $\mathrm{P}+\mathrm{T}-\mathrm{KY} 1$ progeny and their corresponding wild-type tobacco variety in the field at the research station in Oxford, North Carolina (Fig. 5 A-C and Figs S15-S18). Tobacco leaves were air-cured in a contained ventilation barn (Fig. 5 D, Figs S18-S19). 
627 Quantification with GC-FID showed the significant reduction of nicotine, nornicotine, 628 anabasine, anatabine, and myosmine in most or all cured leaves of red P+T-NL1 and $629 \mathrm{P}+\mathrm{T}-\mathrm{KY} 1$ tobacco plants compared to those of non-transgenic controls. The contents 630 of nicotine were significantly reduced by $45-51 \%$ in all cured leaf groups of P+T-NL1 631 (Fig. 6 A) and by 19-30\% in upper three groups of cured leaves of P+T-KY1 (Fig. 6 B). The contents of nornicotine were significantly decreased by $39-44 \%$ and $30-40 \%$

633 in all cured leaf groups of T-P-NL1 and P+T-KY1, respectively (Fig. $6 \mathrm{C}$ and D). The contents of anabasine, anatabine, and myosmine were significantly reduced by $27-$ 45\%, 55-66\%, and 19-25\% in all cured leaf groups of P+T-NL1 (Fig. 6 E, G, and I), respectively, and were significantly reduced by $27-45 \%, 3256 \%$, and $29-35 \%$ in all cured leaf groups of P+T-KY1, respectively (Fig. 6 F, H, and J).

Quantification with HPLC-QQQ-MS/MS showed that the contents of four carcinogenic TSNAs were significantly diminished in most or all leaf groups of $\mathrm{P}+\mathrm{TNL} 1$ and $\mathrm{P}+\mathrm{T}-\mathrm{KY} 1$ plants. The content of NNN was significantly decreased by $63-74 \%$ to a level of $0.136-0.375 \mathrm{ppm}$ in all four groups of cured P+T-NL1 leaves and by $67-79 \%$ to a level of $0.208-0.552 \mathrm{ppm}$ in three groups of cured P+T-KY1 leaves (Fig. 7 A and B). The content of NNN in the B3 leaf group of $\mathrm{P}+\mathrm{T}-\mathrm{KY} 1$ was reduced by $63 \%$ to $0.488 \mathrm{ppm}$ with a slight significance. The content of NNK was reduced by $38-72 \%$ to a level of $0.1-0.173 \mathrm{ppm}$ and by $30-74 \%$ to a level of $0.151-0.3 \mathrm{ppm}$ in four groups of cured P+T-NL1 and P+T-KY1 leaves, respectively, in which the reduction in three leaf groups of each genotype was significant (Fig. $7 \mathrm{C}$ and D). The content of NAT was significantly decreased by $77-92 \%$ to a level of $0.093-0.159 \mathrm{ppm}$ and by $70-80 \%$ to a level of $0.159-0.285$ ppm in all leaf groups of P+T-NL1 and P+T- 
651 KY1, respectively (Fig. $7 \mathrm{E}$ and F). The content of NAB was significantly decreased

652 by $72-80 \%$ to a level of $0.011-0.021 \mathrm{ppm}$ and by $65-77 \%$ to a level of $0.015-0.031$

653 ppm in all leaf groups of P+T-NL1 and P+T-KY1, respectively (Fig. $7 \mathrm{G}$ and H).

654 Furthermore, the contents of NNN, NNK, NAT, and NAB were summed to obtain the 655 total TSNA contents in each leaf group. The total contents of four TSNAs in each 656 group of wild type NL and KY171 leaves were 1.6-2.2 ppm and 2.2-2.6 ppm, while 657 these values were significantly reduced to be $0.4-0.6 \mathrm{ppm}$ and $0.6-1.0 \mathrm{ppm}$ in each 658 group of P+T-NL1 and P+T-KY1 leaves (Fig. 7 I-J). The average contents of four 659 TSNAs in the entire plant leaves of wild type NL and KY171 were approximately $1.77 \mathrm{ppm}$ and $2.25 \mathrm{ppm}$, while these values were significantly reduced to be $0.69 \mathrm{ppm}$ and $0.87 \mathrm{ppm}$ in those of P+T-NL1 and P+T-KY1 (Fig. 7 K-L).

662

663 Furthermore, the total contents of tobacco alkaloids in each group of leaves were 664 summed from nicotine, four other alkaloids, and total TSNAs. The resulting data showed that the contents of total alkaloids were significantly decreased by $44-51 \%$ in four leaf groups of P+TNL1 and 22-35\% in the upper three group leaves of P+T-KY1 (Fig. S20 A-B).

Field trials and reduction of nicotine, nornicotine, anatabine, anabasine, myosmine, and four TSNAs in flue-cured leaves of the red PAP1 tobacco

672 Our red PAP1 tobacco genotype (Fig. S22 B) is a homozygous red variety generated

673 from Xanthi by the overexpression of PAP1 [21]. Gene expression analysis showed 674 that the expression of PAP1 upregulated NtAnla and NtAn1b (Fig. S21), two TT8 
675

676

677

678

679

680

681

682

683

684

685

686

687

688

689

690

691

692

693

694

695

696

697

698

homologs [72]. This result indicated that PAPl alone activated tobacco anthocyanin

biosynthesis via upregulating the endogenous $N$. tabacum TT8 (NtTT8) homologs.

Therefore, to substantiate our de novo design, we used this genotype to test whether

PAP1 and activated NtTT8 homologs could reduce nicotine, other tobacco alkaloids, and TSNAs.

Two years' field trials were completed. The field design (Fig. S22 A-B) and cropping management were the same as described above. When leaves were harvested, they were harvested into five groups (Fig. S22 C-D) and then flue-cured in barns (Fig. S22 E). Analysis with HPLC-ESI-MS obtained peak values that showed the reduction of nicotine, nornicotine, anabasine, and anatabine. The levels of nicotine were significantly or slightly reduced by $10-50 \%$ and $5-25 \%$ in each group of leaves in 2011 and in 2012, respectively (Fig. S23 A-B). The levels of nornicotine, anabasine, and anatabine were significantly or slightly reduced in all or most group leaves by 25$70 \%$ and 5-55\%, 30-60\% and 5-20\%, and 5-60\% and 10-40\% in 2011 and 2012, respectively (Fig. S23 A-B). In addition, the total cumulative peak level of each alkaloid was summed from all leaf groups of the entire PAP1 tobacco plant. The resulting data showed that the total cumulative levels of nicotine, nornicotine, anabasine, and anatabine in the entire plant were significantly reduced by $30 \%, 45 \%$, 50\%, and 40\% in 2011 and 10\%, 20\%, 10\%, and 30\% in 2012 respectively (Fig. S24 A-B).

The contents of NNN, NNK, NAB, and NAT were reduced in flue-cured leaf groups (Fig. S25 A-B). The contents of NNN were reduced by $20-60 \%$ and $25-60 \%$ in all leaf 
699

700

701

groups in two years. The average content of NNN was diminished to a level less than $0.5 \mathrm{ppm}$ in most leaf groups (Fig. S25 A-B). The contents of NNK were reduced by 5$50 \%$ in all leaf groups in 2011 and 5-60\% in four of five leaf groups in 2012. The contents of NAB were reduced by $20-70 \%$ and $15-50 \%$ in all leaf groups in two years. The contents of NAT were reduced by $50-65-\%$ and $35-70 \%$ in all leaf groups in two years. The contents of each NNN, NNK, NAB, and NAT were averaged for all leaf groups of each plant. The resulting data showed that the average contents of each TSNA were significantly reduced by 25-60\% (P-value < 0.05) in 2011 and 2012 (Fig. S25 A-B). The contents of four TSNAs were summed for all leaf groups of each plant. The resulting data showed that the total contents of four TSNAs were significantly reduced by $57 \%$ and $43 \%$ (P-value < 0.05 ) in 2011 and 2012, respectively (Fig. S26).

\section{Discussion}

Our findings indicate that cis-regulatory elements and TFs are useful molecular tools for a de novo design to create a novel regulation of plant secondary metabolism. Herein, based on the regulation mechanism elucidated (Fig. 2), we constructively term the designed regulation to be a Distant Pathway-Cross Regulation (DPCR) of PAP1 and TT8. The rationale is that the pathway of nicotine does not exist in Arabidopsis and two anthocyanin TFs are metabolically characterized to be distant from the tobacco alkaloid pathway. The other reason is that our findings are distinct from the general pleotropic effects that have been generally used to explain gene functions without a mechanistic elucidation. This DPCR definition is characterized by the 
723

mechanisms that PAP1, TT8, and their complex formed with WD40 negatively regulate the tobacco alkaloid biosynthesis via activating $N t J A Z$ repressor genes. Accordingly, this definition distinguishes from the master regulatory function of PAP1, TT8, and their MBW complex that activates the anthocyanin biosynthesis in Arabidopsis $[8,14,73,74]$ or red tobacco shown here via directly activating most or late pathway genes, such as DFR, ANS, and 3-GT (Fig. S10). Based on these data, we propose a model to characterize the DPCR created from the de novo design and the regulation of tobacco alkaloid biosynthesis in red tobacco plants (Fig. 8). Given that NtTTG1 is expressed in roots, leaves, and flowers, this model proposes that PAP1 and TT8 likely anchor to the endogenous tobacco NtTTG1 (WD40) to form a heterogeneous MBW complex, which has a dual regulatory function that regulates tobacco metabolism. First, the MBW complex directly activates the anthocyanin biosynthesis (function I), which is intensively elucidated. Second, the MBW complex upregulates four $N t J A Z 1, N t J A Z 3, N t J A Z 7$, and NtJAZ10, which increase NtJAZ repressors (function II). Next, the increased NtJAZs bind to more MYC2 and block the release of this TF. Then, the reduction of free MYC2 results in downregulating $E F R$ and six or seven nicotine pathway genes (Fig. 3). Finally, the biosynthesis of nicotine, OTAs, and four TSNAs is significantly diminished in red tobacco plants (Figs. 4, 6, and 7). In addition, based on the report that AtMYC2 solely regulates the AtJAZ transcription via a feed-back regulation [75], this model proposes that PAP1/TT8 and NtMYC2 co-activate the expression of NtJAZs in red tobacco plants. When JA is absent, PAP1 and TT8 play the main regulatory role to activate the expression of four NtJAZs. To date, in addition to tobacco, PAP1 and its homologs have been introduced to a few crops to engineer anthocyanins for value-added traits, 
such as tomato [22, 23], hop [24], canola [25], and rose [76]. Although whether and how PAP1 and TT8 can regulate non-anthocyanin or non-flavonoid pathways in these plants remains unknown, it was interesting that PAP1 transgenic rose plants were reported to produce more than 6.5-time scent terpenoids [76]. Based on our findings, it is interesting to investigate whether a similar DPCR occurs to increase scent terpenoids in rose in the future. Moreover, different TF families are a large part of plant genomes [77-80], our findings suggest that it is necessary to investigate DPCR events of other TFs to fully understand their regulatory functions. In particular, as shown in our data that carcinogenic TSNAs are significantly reduced in red tobacco plants, investigations of potential DPCR of TFs likely create value-added traits for crop improvement and human health.

This de novo regulation design with PAP1/TT8 TFs and NtJAZs shows a promising application in enhancing efforts for the simultaneous reduction of most harmful tobacco alkaloids. As introduced above, in the past decades, multiple previous studies had been undertaken to develop technologies to reduce harmful tobacco alkaloids, such as gene suppression or silencing technologies with anti-sense or RNAi of pathway genes (Fig. 1 B), including PMT [65], ODC [64], QPT [63], NDM [81], A622 [82], BBL [49], and others [42]. On the one hand, most studies successfully reported the decrease of the contents of nicotine, OTAs, or TSNAs in tobacco plants grown in the greenhouse, growth chambers, or field. On the other hand, these previous successes revealed a challenge that all tobacco alkaloids could not be diminished simultaneously. For example, the decrease of nicotine led to either the increase of anatabine or non-reduction of other tobacco alkaloids [64, 65]. In addition, 
771 the reduction of harmful compounds was relatively limited. For instance, an RNAi of 772 A622 could mainly reduce NNN and total TSNAs [81]. A gene silencing of $B B L$ was 773 reported to mainly decrease the nicotine content [49]. Furthermore, all these successes 774 have showed that the reduction of nicotine and other alkaloids to a practical level requires more studies, such as elucidation of metabolic regulation and unknown genes. As intensively characterized, the regulation of the nicotine biosynthesis is via three cohort layers, the presence/absence of an active JA form, the JA and JAZ signaling pathway, and the release of MYC2 for activation of ERF189 and pathway genes [42, 48, 55] (Fig. 1 B). To date, an important unanswered question is how tobacco plants regulate NtJAZs. In Arabidopsis, the expression of AtJAZs is solely activated by AtMYC2 via a feed-back regulation [75]. Based on this, although NtMYC2 members have not been reported to regulate the transcription of NtJAZs in tobacco plants, it can be postulated that NtMYC2 members may also perform a feedback regulation of NtJAZs (Fig. 8). Our de novo design with PAP1 and TT8 and MRE and G-box elements was used to target four NtJAZs. As designed, PAP1, TT8, PAP1 and TT8 together, and their MBW complex bound and activated promoters of the four NtJAZs (Fig. 2) and the stacked overexpression of PAP1 and TT8 led to the upregulation of four NtJAZs (Fig. 3 A-B and K-L, Fig. S13). As the positive result, the biosynthesis of nicotine was downregulated in red $\mathrm{P}+\mathrm{T}$ genotypic plants (Figs. 3 and 4). The contents of nicotine, four OTAs, and four TSNAs were simultaneously reduced in most or all cured leaf groups and entire plant leaves of P-T-NL, P-T-KY, and PAP1 genotypes (Figs. 6 and 7, Figs. S20). The NNN contents were particularly diminished to a level less than $0.55 \mathrm{ppm}$ in most of red tobacco leaves (Fig. 7 A-B). This low content is significant to human health, because NNN is a severe tobacco 
795

796

797

carcinogen and its content in any smokeless tobacco products is limited to $1.00 \mathrm{pm}$ proposed by FDA. In addition, other TSNAs were reduced to a level lower than 0.5 ppm in most or all leaves of red $\mathrm{P}+\mathrm{T}$ genotypic tobacco plants. To further substantiate the function of this de novo regulation design, we tested our PAP1 red tobacco variety, in which the expression of PAP1 activated two NtTT8 homologs (Fig. S21). Although this variety was created from Xanthi, a low-nicotine and flue-cured cultivar, the two years' field trials showed the reduction of nicotine, OTAs, and TSNAs in PAP1 plants (Figs. S23-24 and S25-26). These data indicate that PAP1 can partner with the endogenous tobacco NtTT8 homologs to form a DPCR to downregulate the nicotine biosynthesis. Taken together, these findings provide a new platform to eliminate tobacco carcinogens to a non-harmful level and to alter plant metabolism in other crop plants for novel traits.

The plant kingdom has a large number of TFs that regulate plant development, structure, responses to stresses, and plant metabolisms. In 2000, when the genome of Arabidopsis thaliana was sequenced [83], gene annotation and sequence analysis revealed more than 1500 TFs (accounting for more than 5\% of annotated genes). Since then, more TFs have been reported from numerous sequenced plant genomes, such as rice $[84,85]$ and soybean[86], and multiple browser servers have been developed to search TF families and their regulatory functions [87-93]. By 2017, 156 plant genomes were sequenced, including 16 from Chlorophytae, one from Charophyta, one from Marchantiophyta, two from Byrophyta, one from Lycopodiophyta, two from Coniferophyta, one from Basa Magnoliophyta, 38 from monocots, and 95 Eudicots. [67]. These 156 genomes together with sequences of nine 
additional plant species allowed the annotation of 320,370 plant TFs, which were grouped into 58 families [67]. Likewise, the plant genomes have a large number of

821 binding sites (motifs) (BS), such as G-box and MRE in promoters, to which TFs bind.

822 In recent, a public PlantRegMap server was developed from 63 plant species (out of

823156 genome-sequenced plants) to search TFBSs and the interactions between TF and

824 BS [68]. This PlantRegMap characterized a conserved regulatory landscape that included 21,997,501 TFBSs, 21,346 TFs, and more than two million interactions between them [68]. It can be speculated that as more plant species will be sequences,

827 the number of plant TFs will be increased. To our knowledge, none of TFs and BSs has been used as molecular tools for a novel regulatory design to engineer better

TFs and BSs provide an extremely rich molecular tool source for novel regulatory designs, which will be fundamental for plant engineering.

\section{Conclusion}

837 The findings show that PAP1, TT8, MREs, and G-box elements are useful molecular 838 tools to design a De Novo regulation, a distant pathway-cross regulation (DPCR) of 839 plant secondary metabolism in plants. The findings unearth novel regulatory functions 840 of PAP1 and TT8 that are two positive regulators of NtJAZs and negatively regulate 841 tobacco alkaloid biosynthesis in red tobacco plants. This De Novo regulation design 842 significantly reduces harmful nicotine, other tobacco alkaloids, and all TSNAs in all 
843 or most leaves of tobacco plants. Our findings indicate that the great number of TFs

844 and BSs provide an extremely rich molecular tool source for novel regulatory designs,

845 which will be fundamental in plant engineering for value-added crops and products.

846

847 Conflict of Interest

848 Authors declare no conflict of interest.

849

850

851 Supporting information

852

853 Supporting materials and growth conditions, Method S1-S8, 26 supporting figures and

8549 supporting tables.

855

85626 Supporting Figure legends

857

858 Figure S1 Promoter sequences of NtJAZ1 and NtJAZ3 and identification of cis-

859 elements

860 Figure S2 Promoter sequences of NtJAZ7 and NtJAZ10 and identification of cis-

861 elements

862 Figure S3 Images showing expression and purification of the binding domains of

863 PAP1 and TT8 induced in E. coli in vitro

864 Figure S4 Promoter sequences of NtPMT2 and NtODC and identification of cis-

865 elements. 
Figure S5 Electrophoretic mobility shift assays (EMSAs) showing weak binding of TT8 to G-box-like probe and no binding of PAP1 to MRE-like probe.

Figure S6 Dual-luciferase and Chip-qPCR experiments showing no activation of NtPMT2 and NtODC2 promoters bound by PAP1 and TT8 alone, PAP1 and TT8 together, and PAP1-TT8-WD complex.

Figure S7 Diagrams showing gene stacking design for DNA synthesis, binary construct, and selection of T2 homozygous plants.

Figure S8 Comparison of AtPAP1 and AtTT8 transgene expressions in roots of wildtype Narrow Leaf Madole (NL) and KY171, P+T transgenic NL and KY171, and vector control transgenic plants.

Figure S9 Phenotypes of wild type, vector-control, and P+T-NL and P+T-KY tobacco plants used for analysis of gene expression and metabolites.

Figure S10 Transcriptional upregulation of eight flavonoid pathway genes in red transgenic $\mathrm{P}+\mathrm{T}-\mathrm{NL}$ and $\mathrm{P}+\mathrm{T}-\mathrm{KY}$ tobacco plants.

Figure S11 Anthocyanin levels in roots and leaves of wild type and red P+T transgenic tobacco plants.

Figure S12 Proanthocyanidin formation in roots of red P+T transgenic tobacco plants Figure S13 Comparison of NtJAZ7 and NtJAZ10 gene expression in roots of wild-type Narrow Leaf Madole (NL) and KY171, P+T transgenic NL and KY171, and vector control transgenic plants.

Figure S14 Comparison of MYC1a, MYC1b, MYC2a and MYC2b gene expression in roots of wild-type (WT) Narrow Leaf Madole (NL) and KY171, P+T transgenic NL and $\mathrm{KY}$, and vector control transgenic plants. 
889

890

891

Figure S15 Schematic diagram showing procedures for field farming practice and leaf harvest from wild type and red transgenic tobacco plants.

Figure S16 Field design for farming practice of red $\mathrm{P}+\mathrm{T}$ transgenic plants.

Figure S17 Planting, plant growth, and phenotypes of wild type and red P+T transgenic tobacco plants in field from the first day to 75 days after planting.

Figure S18 Harvest of plants from field for air-curing and cleanup of field.

Figure S19 Comparison of leaf color after air-curing and grouping of leaves for sampling.

Figure S20 Reduction of total tobacco alkaloids in each leaf group of red P+T-NL1 and $\mathrm{P}+\mathrm{T}-\mathrm{KY} 1$ plants compared to wild type ones.

Figure S21 Upregulation of NtAnla and NtAnlb in roots of red PAP1 tobacco plants.

Figure S22 Field farming practice of PAP1 tobacco plants and leaf harvest.

Figure S23 Reduction of nicotine, nornicotine, anabasine, and anatabine in PAP1 tobacco leaves.

Figure S24 Reduction of total nicotine, nornicotine, anabasine, and anatabine in all leaves of PAP1 tobacco plant.

Figure S25 Reduction of NNN, NNK, NAB, and NAT in flue-cured leaves of PAP1 tobacco compared to leaves of wild type Xanthi control.

Figure S26 Reduction of total TSNAs in flue-cured leaves of PAP1 tobacco.

\section{Nine Supporting Table capitals}

Table S1 Thermal cycle and sequences of primers designed for PCR, qRT-PCR, QPCR, respectively. 
912 Table S2 Float trays used for seedlings in the water nursery bed in the greenhouse.

913 The same trays were prepared for PAP1 tobacco plants.

914 Table S3 Instrument Parameters used in Tobacco Specific Nitrosamines analysis.

915 Table S4 HPLC Gradient used in Tobacco Specific Nitrosamines analysis.

916 Table S5 Typical Parameter Table Triple Quadrupole Mass Spectrometer used in

917 Tobacco Specific Nitrosamines analysis.

918 Table S6 Reagents and Standards in for Tobacco Specific Nitrosamines analysis.

919 Table S7 Instrument Parameters used in Tobacco Alkaloids analysis.

920 Table S8 Oven Program used for in Tobacco Alkaloids analysis.

921 Table S9 Reagents and Standards used for Tobacco Alkaloids analysis.

922 

Transcription Factor Gene Family in Maize, PLoS One 7(6) (2012) 12. [2] C. Dubos, R. Stracke, E. Grotewold, B. Weisshaar, C. Martin, L. Lepiniec, MYB transcription factors in Arabidopsis, Trends Plant Sci 15(10) (2010) 573-581.

[3] A. Feller, K. Machemer, E.L. Braun, E. Grotewold, Evolutionary and comparative analysis of MYB and bHLH plant transcription factors, Plant J. 66(1) (2011) 94-116.

[4] K. Wei, H. Chen, Comparative functional genomics analysis of bHLH gene family in rice, maize and wheat, BMC Plant Biol. 18(1) (2018) 309.

[5] T. Saigo, T. Wang, M. Watanabe, T. Tohge, Diversity of anthocyanin and proanthocyanin biosynthesis in land plants, Curr. Opin. Plant Biol. 55 (2020) 93-99. [6] M.-Z. Shi, D.-Y. Xie, Biosynthesis and metabolic engineering of anthocyanins in Arabidopsis thaliana, Recent Pat Biotechnol 8(1) (2014) 47-60.

[7] K. Springob, H. Nakajima, M. Yamazaki, K. Saito, Recent advances in the biosynthesis and accumulation of anthocyanins, Nat Prod Rep 20 (2003) 288-303. [8] J.O. Borevitz, Y. Xia, J. Blount, R.A. Dixon, C. Lamb, Activation tagging identifies a conserved MYB regulator of phenylpropanoid biosynthesis, Plant Cell 12(12) (2000) 2383-2394.

[9] N. Nesi, I. Debeaujon, C. Jond, G. Pelletier, M. Caboche, L. Lepiniec, The TT8 gene encodes a basic helix-loop-helix domain protein required for expression of $D F R$ and BAN genes in Arabidopsis siliques, The Plant Cell 12 (2000) 1863-1878. [10] A.R. Walker, P.A. Davison, A.C. Bolognesi-Winfield, C.M. James, N. Srinivasdan, T.L. Blundell, J.J. Esch, M.D. Marks, J.C. Gray, The TRANSPARENT TESTA GLABRA 1 locus, which regulates trichome differentiation and anthocyanin biosynthesis in Arabidopsis, encodes a WD40 repeat protein, Plant Cell 11 (1999) 1377-1349.

[11] W. Xu, D. Grain, S. Bobet, J. Le Gourrierec, J. Thévenin, Z. Kelemen, L. Lepiniec, C. Dubos, Complexity and robustness of the flavonoid transcriptional regulatory network revealed by comprehensive analyses of MYB-bHLH-WDR complexes and their targets in Arabidopsis seed, New Phytol. 202(1) (2014) 132-144. [12] J. Thévenin, C. Dubos, W. Xu, J. Le Gourrierec, Z. Kelemen, F. Charlot, F. Nogué, L. Lepiniec, B. Dubreucq, A new system for fast and quantitative analysis of heterologous gene expression in plants, New Phytol. 193(2) (2012) 504-512.

[13] W.J. Xu, D. Grain, J. Le Gourrierec, E. Harscoet, A. Berger, V. Jauvion, A. Scagnelli, N. Berger, P. Bidzinski, Z. Kelemen, F. Salsac, A. Baudry, J.M. Routaboul, L. Lepiniec, C. Dubos, Regulation of flavonoid biosynthesis involves an unexpected complex transcriptional regulation of TT8 expression, in Arabidopsis, New Phytol. 198(1) (2013) 59-70.

[14] Z.X. Zhu, H.L. Wang, Y.T. Wang, S. Guan, F. Wang, J.Y. Tang, R.J. Zhang, L.L. Xie, Y.Q. Lu, Characterization of the cis elements in the proximal promoter regions of the anthocyanin pathway genes reveals a common regulatory logic that governs pathway regulation, J. Exp. Bot. 66(13) (2015) 3775-3789.

[15] A. Baudry, M.A. Heim, B. Dubreucq, M. Caboche, B. Weisshaar, L. Lepiniec, TT2, TT8, and TTG1 synergistically specify the expression of BANYULS and proanthocyanidin biosynthesis in Arabidopsis thaliana, Plant J 39(3) (2004) 366-380. 
[16] N.A. Ramsay, B.J. Glover, MYB-bHLH-WD40 protein complex and the evolution of cellular diversity, Trends Plant Sci 10(2) (2005) 63-70.

[17] M.Z. Shi, D.Y. Xie, Engineering of red cells of Arabidopsis thaliana and comparative genome-wide gene expression analysis of red cells versus wild-type cells, Planta 233(4) (2011) 787-805.

[18] T. Qi, S. Song, Q. Ren, D. Wu, H. Huang, Y. Chen, M. Fan, W. Peng, C. Ren, D. Xie, The jasmonate-ZIM-domain proteins interact with the WD-Repeat/bHLH/MYB complexes to regulate jasmonate-mediated anthocyanin accumulation and trichome initiation in Arabidopsis thaliana, Plant Cell 23(5) (2011) 1795-1814. [19] T.C. Qi, H. Huang, D.W. Wu, J.B. Yan, Y.J. Qi, S.S. Song, D.X. Xie, Arabidopsis DELLA and JAZ proteins bind the WD-Repeat/bHLH/MYB complex to modulate gibberellin and jasmonate signaling synergy, Plant Cell 26(3) (2014) 11181133.

[20] X. He, Y. Li, D. Lawson, D.-Y. Xie, Metabolic engineering of anthocyanins in dark tobacco varieties Physiol Plant 159 (2017) 2-12.

[21] D.-Y. Xie, S.B. Sharma, E. Wright, Z.-Y. Wang, R.A. Dixon, Metabolic engineering of proanthocyanidins through co-expression of anthocyanidin reductase and the PAP1 MYB transcription factor, Plant J 45(6) (2006) 895-907. [22] E. Butelli, L. Titta, M. Giorgio, H.-P. Mock, A. Matros, S. Peterek, E.G.W.M. Schijlen, R.D. Hall, A.G. Bovy, J. Luo, C. Martin, Enrichment of tomato fruit with health-promoting anthocyanins by expression of select transcription factors, Nat Biotech 26(11) (2008) 1301-1308. [23] D.L. Zuluaga, S. Gonzali, E. Loreti, C. Pucciariello, E.D. Degl'Innocenti, L. Guidi, A. Alpi, P. Perata, Arabidopsis thaliana MYB75/PAP1 transcription factor induces anthocyanin production in transgenic tomato plants, Funct Plant Biol 35(7) (2008) 606-618.

[24] A. Gatica-Arias, M.A. Farag, M. Stanke, J. Matousek, L. Wessjohann, G. Weber, Flavonoid production in transgenic hop (Humulus lupulus L.) altered by PAP1/MYB75 from Arabidopsis thaliana L, Plant Cell Rep 31(1) (2012) 111-119. [25] X. Li, M.J. Gao, H.Y. Pan, D.J. Cui, M.Y. Gruber, Purple canola: Arabidopsis PAP1 increases antioxidants and phenolics in Brassica napus leaves, J Agric Food Chem 58(3) (2010) 1639-1645.

[26] D.M. Ma, S.V.S. Gandra, R. Manoharlal, C. La Hovary, D.Y. Xie, Untargeted metabolomics of Nicotiana tabacum grown in United States and India characterizes the association of plant metabolomes wiith natural climate and geography, Front. Plant Sci. 10 (2019) 18.

[27] L.H. Nugroho, R. Verpoorte, Secondary metabolism in tobacco, Plant Cell, Tissue and Organ Culture 68(2) (2002) 105-125. [28] A. Rodgman, T.A. Perfetti, The Chemical Components of Tobacco and Tobacco Smoke, CRC Press, Taylor \& Francis Group, Boca Raton, London, New York, 2009. [29] I. Arany, M. Taylor, T. Fulop, M. Dixit, Adverse effects of chronic nicotine exposure on the kidney: Potential human health implications of experimental findings, International Journal of Clinical Pharmacology and Therapeutics 56(11) (2018) 501506.

[30] S.A. Grando, Connections of nicotine to cancer, Nature Reviews Cancer 14(6) (2014) 419-429. 
bioRxiv preprint doi: https://doi.org/10.1101/2020.12.20.423678; this version posted June 22, 2021. The copyright holder for this preprint (which was not certified by peer review) is the author/funder. All rights reserved. No reuse allowed without permission.

[31] L. Greillier, A.B. Cortot, J. Viguier, L. Brignoli-Guibaudet, C. Lhomel, F. Eisinger, J.F. Morere, S. Couraud, Perception of Lung Cancer Risk: Impact of Smoking Status and Nicotine Dependence, Current Oncology Reports 20 (2018) 7. [32] P.N. Lee, B.A. Forey, J.S. Hamling, A.J. Thornton, Environmental tobacco smoke exposure and heart disease: A systematic review, World Journal of MetaAnalysis 5(2) (2017) 14-40.

[33] T. Sanner, T.K. Grimsrud, Nicotine: carcinogenicity and effects on response to cancer treatment - a review, Frontiers in Oncology 5 (2015) 10.

[34] A. Santoro, C. Tomino, G. Prinzi, P. Lamonaca, V. Cardaci, M. Fini, P. Russo, Tobacco Smoking: Risk to Develop Addiction, Chronic Obstructive Pulmonary Disease, and Lung Cancer, Recent Patents on Anti-Cancer Drug Discovery 14(1) (2019) 39-52.

[35] A.N. Thorndike, N.A. Rigotti, A tragic triad: coronary artery disease, nicotine addiction, and depression, Current Opinion in Cardiology 24(5) (2009) 447-453. [36] J.W. Tidey, D.R. Davis, M.E. Miller, I. Pericot-Valverde, R.L. Denlinger-Apte, D.E. Gaalema, Modeling nicotine regulation: A review of studies in smokers with mental health conditions, Prev. Med. 117 (2018) 30-37.

[37] S.S. Hecht, Biochemistry, biology, and carcinogenicity of tobacco-specific Nnitrosamines, Chemical Research in Toxicology 11(6) (1998) 559-603.

[38] S.S. Hecht, I. Stepanov, S.G. Carmella, Exposure and Metabolic Activation Biomarkers of Carcinogenic Tobacco-Specific Nitrosamines, Accounts of Chemical Research 49(1) (2016) 106-114.

[39] E. Konstantinou, F. Fotopoulou, A. Drosos, N. Dimakopoulou, Z. Zagoriti, A. Niarchos, D. Makrynioti, D. Kouretas, K. Farsalinos, G. Lagoumintzis, K. Poulas, Tobacco-specific nitrosamines: A literature review, Food Chem. Toxicol. 118 (2018) 198-203.

[40] FDA, Tobacco Product Standard for N-Nitrosonornicotine Level in Finished Smokeless Tobacco Products, in: H.A.H. SERVICES (Ed.) Food and Drug Administration, 2017.

[41] R.F. Dawson, An experimental analysis of alkaloid production in Nicotiana - The origin of nornicotine, Am J Bot 32(7) (1945) 416-423.

[42] R.E. Dewey, J.H. Xie, Molecular genetics of alkaloid biosynthesis in Nicotiana tabacum, Phytochemistry 94 (2013) 10-27.

[43] B. Ladesic, T.C. Tso, Biochemical studies on tobacco alkaloids. 6. Biosynthesis of nicotine through nornicotine, Phytochemistry 3(4) (1964) 541-545.

[44] S. Mizusaki, Y. Tanabe, M. Noguchi, E. Tamaki, Phytochemical studies on tobacco alkaloids. 16. Changes in activities of ornitheine decarboxylase, putrescine Nmethyltransferase and $\mathrm{N}$-methyl-putrescine oxidase in tobacco roots in relation to nicotine biosynthesis, Plant Cell Physiol 14(1) (1973) 103-110.

[45] W. Stepka, L.J. Dewey, Conversion of nicotine to nornicotine in harvested tobacco - fate of methyl group, Plant Physiol 36(5) (1961) 592-\&.

[46] T.C. Tso, R.N. Jeffrey, Studies on tobacco alkaloids. 1. Changes in nicotine and nornicotine content in Nicotiana, Plant Physiol 31(6) (1956) 433-439.

[47] T.C. Tso, R.N. Jeffrey, Studies on tobacco alkaloids. 2. The formation of nicotine and nornicotine in tobacco supplied with N15, Plant Physiol 32(2) (1957) 86-92.

[48] T. Shoji, T. Hashimoto, Smoking out the masters: transcriptional regulators for nicotine biosynthesis in tobacco, Plant Biotechnol 30(3) (2013) 217-224. 
1062

1063

1064

1065

1066

1067

1068

1069

1070

1071

1072

1073

1074

1075

1076

1077

1078

1079

1080

1081

1082

1083

1084

1085

1086

1087

1088

1089

1090

1091

1092

1093

1094

1095

1096

1097

1098

1099

1100

1101

1102

1103

1104

1105

1106

1107

1108

[49] R.S. Lewis, H.O. Lopez, S.W. Bowen, K.R. Andres, W.T. Steede, R.E. Dewey, Transgenic and mutation-based suppression of a berberine bridge enzyme-like (BBL) gene family reduces alkaloid content in field-grown tobacco, PLoS One 10(2) (2015). [50] M. Chakrabarti, S.W. Bowen, N.P. Coleman, K.M. Meekins, R.E. Dewey, B. Siminszky, CYP82E4-mediated nicotine to nornicotine conversion in tobacco is regulated by a senescence-specific signaling pathway, Plant Mol Biol 66(4) (2008) 415-427.

[51] L.B. Gavilano, B. Siminszky, Isolation and characterization of the cytochrome P450 gene CYP82E5v2 that mediates nicotine to nornicotine conversion in the green leaves of tobacco, Plant Cell Physiol 48(11) (2007) 1567-1574.

[52] R.S. Lewis, S.W. Bowen, M.R. Keogh, R.E. Dewey, Three nicotine demethylase genes mediate nornicotine biosynthesis in Nicotiana tabacum L.: Functional characterization of the CYP82E10 gene, Phytochemistry 71(17-18) (2010) 19881998.

[53] K. De Boer, S. Tilleman, L. Pauwels, R. Vanden Bossche, V. De Sutter, R. Vanderhaeghen, P. Hilson, J.D. Hamill, A. Goossens, APETALA2/ETHYLENE RESPONSE FACTOR and basic helix-loop-helix tobacco transcription factors cooperatively mediate jasmonate-elicited nicotine biosynthesis, Plant J 66(6) (2011) 1053-1065.

[54] T. Shoji, T. Hashimoto, Tobacco MYC2 regulates jasmonate-inducible nicotine biosynthesis genes directly and by way of the NIC2-locus ERF genes, Plant Cell Physiol 52(6) (2011) 1117-1130.

[55] H.B. Zhang, M.T. Bokowiec, P.J. Rushton, S.C. Han, M.P. Timko, Tobacco transcription factors NtMYC2a and NtMYC2b form nuclear complexes with the NtJAZ1 repressor and regulate multiple jasmonate-inducible steps in nicotine biosynthesis, Mol Plant 5(1) (2012) 73-84.

[56] I.T. Baldwin, Z.P. Zhang, N. Diab, T.E. Ohnmeiss, E.S. McCloud, G.Y. Lynds, E.A. Schmelz, Quantification, correlations and manipulations of wound-induced changes in jasmonic acid and nicotine in Nicotiana sylvestris, Planta 201 (1997) 397404.

[57] S. Imanishi, K. Hashizume, M. Nakakita, H. Kojima, Y. Matsubayashi, T. Hashimoto, Y. Sakagami, Y. Yamada, K. Nakamura, Differential induction by methyl jasmonate of genes encoding ornithine decarboxylase and other enzymes involved in nicotine biosynthesis in tobacco cell cultures, Plant Mol Biol 38(6) (1998) 1101-1111. [58] T. Shoji, Y. Yamada, T. Hashimoto, Jasmonate induction of putrescine Nmethyltransferase genes in the root of Nicotiana sylvestris, Plant Cell Physiol 41(7) (2000) 831-839.

[59] B.F. Xu, M.P. Timko, Methyl jasmonate induced expression of the tobacco putrescine N-methyltransferase genes requires both G-box and GCC-motif elements, Plant Mol Biol 55(5) (2004) 743-761.

[60] T. Shoji, T. Ogawa, T. Hashimoto, Jasmonate-induced nicotine formation in tobacco is mediated by tobacco COI1 and JAZ genes, Plant Cell Physiol 49(7) (2008) 1003-1012.

[61] H.Y. Zhang, W.J. Li, D.X. Niu, Z.J. Wang, X.X. Yan, X.L. Yang, Y.F. Yang, H. Cui, Tobacco transcription repressors NtJAZ: Potential involvement in abiotic stress response and glandular trichome induction, Plant Physiol. Biochem. 141 (2019) 388397. 
[62] K.D. DeBoer, H.L. Dalton, F.J. Edward, S.M. Ryan, J.D. Hamill, RNAimediated down-regulation of ornithine decarboxylase (ODC) impedes wound-stress stimulation of anabasine synthesis in Nicotiana glauca, Phytochemistry 86 (2013) 2128.

[63] J. Xie, S. W, W. Maksymowicz, J. W, C. K, C. Wx, C. C, K. J, C. Ma, Biotechnology: A tool for reduced risk tobacco products - The nicotine experience from test tube to cigarette pack, Recent Advances in Tobacco Science, 2004, pp. 1737.

[64] K.D. DeBoer, H.L. Dalton, F.J. Edward, J.D. Hamill, RNAi-mediated downregulation of ornithine decarboxylase (ODC) leads to reduced nicotine and increased anatabine levels in transgenic Nicotiana tabacum L, Phytochemistry 72(4-5) (2011) 344-355.

[65] Y. Chintapakorn, J.D. Hamill, Antisense-mediated down-regulation of putrescine $\mathrm{N}$-methyltransferase activity in transgenic Nicotiana tabacum L.can lead to elevated levels of anatabine at the expense of nicotine, Plant Mol.Biol. 53 (2003) 87-105. [66] J.L. Riechmann, J. Heard, G. Martin, L. Reuber, C.Z. Jiang, J. Keddie, L. Adam, O. Pineda, O.J. Ratcliffe, R.R. Samaha, R. Creelman, M. Pilgrim, P. Broun, J.Z. Zhang, D. Ghandehari, B.K. Sherman, G.L. Yu, Arabidopsis transcription factors: genome-wide comparative analysis among Eukaryotes, Science 290 (2000) 2105 2110.

[67] J. Jin, F. Tian, D.-C. Yang, Y.-Q. Meng, L. Kong, J. Luo, G. Gao, PlantTFDB 4.0: toward a central hub for transcription factors and regulatory interactions in plants, Nucleic Acids Res 45(D1) (2016) D1040-D1045. [68] F. Tian, D.-C. Yang, Y.-Q. Meng, J. Jin, G. Gao, PlantRegMap: charting functional regulatory maps in plants, Nucleic Acids Res 48(D1) (2019) D1104D1113.

[69] M.Z. Li, Y.Z. Li, L.L. Guo, N.D. Gong, Y.Z. Pang, W.B. Jiang, Y.J. Liu, X.L. Jiang, L. Zhao, Y.S. Wang, D.Y. Xie, L.P. Gao, T. Xia, Functional characterization of tea (Camellia sinensis) MYB4a transcription factor using an integrative approach, Front. Plant Sci. 8 (2017) 17.

[70] P. Wang, Y. Liu, L. Zhang, W. Wang, H. Hou, Y. Zhao, X. Jiang, J. Yu, H. Tan, Y. Wang, D.-Y. Xie, L. Gao, T. Xia, Functional demonstration of plant flavonoid carbocations proposed to be involved in the biosynthesis of proanthocyanidins, The Plant Journal 101(1) (2020) 18-36.

[71] D.-Y. Xie, S.B. Sharma, N.L. Paiva, D. Ferreira, R.A. Dixon, Role of anthocyanidin reductase, encoded by BANYULS in plant flavonoid biosynthesis, Science 299(5605) (2003) 396-399.

[72] Y.H. Bai, S. Pattanaik, B. Patra, J.R. Werkman, C.H. Xie, L. Yuan, Flavonoidrelated basic helix-loop-helix regulators, NtAn1a and NtAn1b, of tobacco have originated from two ancestors and are functionally active, Planta 234(2) (2011) 363375.

[73] A. Baudry, M. Caboche, L. Lepiniec, TT8 controls its own expression in a feedback regulation involving TTG1 and homologous MYB and bHLH factors, allowing a strong and cell-specific accumulation of flavonoids in Arabidopsis thaliana, Plant J 46(5) (2006) 768-779.

[74] A. Gonzalez, M. Zhao, J.M. Leavitt, A.M. Lloyd, Regulation of the anthocyanin biosynthetic pathway by the TTG1/bHLH/Myb transcriptional complex in Arabidopsis seedlings, Plant J 53(5) (2008) 814-827. 
1157

1158

1159

1160

1161

1162

1163

1164

1165

1166

1167

1168

1169

1170

1171

1172

1173

1174

1175

1176

1177

1178

1179

1180

1181

1182

1183

1184

1185

1186

1187

1188

1189

1190

1191

1192

1193

1194

1195

1196

1197

1198

1199

1200

1201

1202

[75] A. Chini, S. Fonseca, G. Fernandez, B. Adie, J.M. Chico, O. Lorenzo, G. GarciaCasado, I. Lopez-Vidriero, F.M. Lozano, M.R. Ponce, J.L. Micol, R. Solano, The JAZ family of repressors is the missing link in jasmonate signalling, Nature 448(7154) (2007) 666-+.

[76] M.M.B. Zvi, E. Shklarman, T. Masci, H. Kalev, T. Debener, S. Shafir, M. Ovadis, A. Vainstein, PAP1 transcription factor enhances production of phenylpropanoid and terpenoid scent compounds in rose flowers, New Phytol. (2012) 10.1111/j.1469-8137.2012.04161.x.

[77] K. Feng, X.L. Hou, G.M. Xing, J.X. Liu, A.Q. Duan, Z.S. Xu, M.Y. Li, J. Zhuang, A.S. Xiong, Advances in AP2/ERF super-family transcription factors in plant, Critical reviews in biotechnology 40(6) (2020) 750-776.

[78] P.S. Millard, B.B. Kragelund, M. Burow, R2R3 MYB transcription factors functions outside the DNA-binding domain, Trends Plant Sci 24(10) (2019) 934-946. [79] M. Pireyre, M. Burow, Regulation of MYB and bHLH transcription factors: a glance at the protein level, Mol Plant 8(3) (2015) 378-88.

[80] M.K. Skinner, A. Rawls, J. Wilson-Rawls, E.H. Roalson, Basic helix-loop-helix transcription factor gene family phylogenetics and nomenclature, Differentiation; research in biological diversity 80(1) (2010) 1-8.

[81] R.S. Lewis, A.M. Jack, J.W. Morris, V.J.M. Robert, L.B. Gavilano, B. Siminszky, L.P. Bush, A.J. Hayes, R.E. Dewey, RNA interference (RNAi)-induced suppression of nicotine demethylase activity reduces levels of a key carcinogen in cured tobacco leaves, Plant Biotechnol J 6(4) (2008) 346-354.

[82] K.D. DeBoer, J.C. Lye, C.D. Aitken, A.K.K. Su, J.D. Hamill, The A622 gene in Nicotiana glauca (tree tobacco): evidence for a functional role in pyridine alkaloid synthesis, Plant Mol Biol 69(3) (2009) 299-312.

[83] T.A.G. Initiative, Analysis of the genome sequence of the flowering plant Arabidopsis thaliana, Nature 408 (2000) 796-815.

[84] J. Yu, e. al., A draft sequuence of the rice genome (Oryza sativa L. ssp indica), Science 296 (2002) 79-92.

[85] S.A. Goff, e. al, A draft sequence of the rice genome (Oryza sativa L. ssp japonica), Science 296 (2002) 92-100.

[86] M.Y. Kim, S. Lee, K. Van, T.H. Kim, S.C. Jeong, I.Y. Choi, D.S. Kim, Y.S. Lee, D. Park, J. Ma, W.Y. Kim, B.C. Kim, S. Park, K.A. Lee, D.H. Kim, K.H. Kim, J.H. Shin, Y.E. Jang, K. Do Kim, W.X. Liu, T. Chaisan, Y.J. Kang, Y.H. Lee, J.K. Moon, J. Schmutz, S.A. Jackson, J. Bhak, S.H. Lee, Whole-genome sequencing and intensive analysis of the undomesticated soybean (Glycine soja Sieb. and Zucc.) genome, PNAS 107(51) (2010) 22032-22037.

[87] A.Y. Guo, X. Chen, G. Gao, H. Zhang, Q.H. Zhu, X.C. Liu, Y.F. Zhong, X.C. $\mathrm{Gu}, \mathrm{K}$. He, J.C. Luo, PlantTFDB: a comprehensive plant transcription factor database, Nucleic Acids Res 36 (2008) D966-D969.

[88] J. Jin, H. Zhang, L. Kong, G. Gao, J. Luo, PlantTFDB 3.0: a portal for the functional and evolutionary study of plant transcription factors, Nucleic Acids Res 42(D1) (2013) D1182-D1187.

[89] A.Y. Guo, K. He, D. Liu, S.N. Bai, X.C. Gu, L.P. Wei, J.C. Luo, DATF: a database of Arabidopsis transcription factors, Bioinformatics 21(10) (2005) 25682569. 
[90] K. Iida, M. Seki, T. Sakurai, M. Satou, K. Akiyama, T. Toyoda, A. Konagaya, K. Shinozaki, RARTF: Database and tools for complete sets of Arabidopsis transcription factors, DNA Res. 12(4) (2005) 247-256.

[91] G. Gao, Y.F. Zhong, A.Y. Guo, Q.H. Zhu, W. Tang, W.M. Zheng, X.C. Gu, L.P. Wei, J.C. Luo, DRTF: a database of rice transcription factors, Bioinformatics 22(10) (2006) 1286-1287.

[92] D.M. Riano-Pachon, S. Ruzicic, I. Dreyer, B. Mueller-Roeber, PlnTFDB: an integrative plant transcription factor database, BMC Bioinformatics 8 (2007) 10. [93] J. Fredslund, DATFAP: A database of primers and homology alignments for transcription factors from 13 plant species, Bmc Genomics 9 (2008) 10.

\section{Figure Legends}

\section{Figure 1 Biosynthetic pathways of nicotine, other tobacco alkaloids, and}

anthocyanin and their regulations. A, the biosynthetic pathway of Arabidopsis

anthocyanin from the step catalyzed by CHS. PAP1, TT8, and TTG1 encode MYB75, bHLH, and WD40 to form a MYB-bHLH-WD40 complex that activates late pathway genes. Gene abbreviations are $C H S$ and $C H I$ : chalcone synthase and isomerase, $F 3 H$ : flavonone-3-hydroxylase, $F 3$ ' $H$ and $F 3$ '5' $H$ : flavonoid-3'-hydroxylase and 3'5'hydroxylase, DFR: dihydroflavonol reductase, ANS: anthocyanidin synthase, 3-

GT: glycosyltransferase. ANR encodes anthocyanidin reductase, a key enzyme toward proanthocyanidin biosynthesis. B, the biosynthetic pathway of nicotine, nornicotine, and tobacco specific nitrosamines (TSNAs) and the regulation mechanism. MYC2 and EAR are two positive transcription factors that activate the expression of most pathway genes. In the absence of isoleucine-jasmonate (Ile-JA), NtJAZ1 and NtJAZ3 repressors bind to MYC2 to inhibit the activation of the pathway genes (red lines). In the presence of bioactive Ile-JA (green arrows), $\mathrm{SCF}^{\mathrm{COI} 1}$ perceives this plant hormone to form a new JA-SCF ${ }^{\mathrm{COI} 1}$ complex to bind to NtJAZs and lead them to proteasomal 
1232

1233

1234

1235

1236

1237

1238

1239

1240

1241

1242

1243

1244

1245

1246

1247

1248

1249

1250

1251

1252

1253

1254

1255

ubiquitination. The degradation of NtJAZs releases MYC2 to activate pathway gene expression toward the biosynthesis of nicotine. Gene abbreviations are $A O$ : aspartate oxidase, $Q S$ : quinolinate synthase, $Q P T 2$ : quinolinate phosphoribosyltransferase 2, $A D C$ : arginine decarboxylase, $A I H$ : agmatine iminohydrolase, $O D C$ : ornithine decarboxylase, MPT1: N-methylputricine transferase 1, MPO1: methylputricine oxidase 1, $A 622$ : an isoflavone reductase like enzyme, $B B L$ : berberine bridge enzymelike protein, and $N N D$ : nicotine $\mathrm{N}$-demethylase.

\section{Figure 2 Binding and activation of $N t J A Z 1$ and $N t J A Z 3$ promoters by PAP1 and} TT8 alone, PAP1 and TT8 together, and PAP1-TT8-WD40 complex. A, three MYB response element (MRE) types and G-box were identified in the NtJAZ1 and NtJAZ3 promoters; B, electrophoretic mobility shift assay (EMSA) showed that PAP1 and TT8 bound to three MRE types and G-box of the NtJAZ1 and NtJAZ3 promoters, respectively. Competitive and non-competitive probes were used for binding of both TFs. 20x and 10x: concentrations of tested competitive and non-competitive probes 20 and 10 times as those MRE and G-box probes. C, fold change values from ChIPqPCR showed that both PAP1 and TT8 bound to the NtJAZ1 and NtJAZ3 promoters in vivo. Approximately $150 \mathrm{bp} N t J A Z 1$ and NtJAZ3 promoter fragments containing both MRE and G-box were enriched with anti-HA antibodies in qRT-PCR analysis. The regions of $N t J A Z 1$ and $N t J A Z 3$ promoters that do not contain a MRE and G-box were used as negative controls. Green and red bars represent wild type and red tobacco plants, respectively. D, schematic diagrams shows four effector constructs (PK2GW7GFP, PAP1, TT8 and TTG1) and two reporter constructs (pGreenII-0800-Jaz1pro and Jaz3 pro) used for dual-luciferase assay. E, luciferase (LUC)/renilla (REN) 
1256

1257

1258

1259

1260

1261

1262

1263

1264

1265

1266

1267

1268

1269

1270

1271

1272

1273

1274

1275

1276

1277

1278

1279

luminescence ratios from dual luciferase assays showed that PAP1 and TT8 alone bound to and activated the promoters of both NtJAZ1 and NtJAZ3, two TFs together increased promoter activity, and two TFs and WD40 together increased the highest activity. The promoters were fused to a firefly luciferase (reporter). The promoters of NtJAZ1 and NtJAZ3 genes were used in dual luciferase assay. Values represent the mean \pm S.D. $(n=5)$. Asterisks on top of bars indicate that the values are significantly higher than those of controls $(* \mathrm{P}<0.05$, $* * \mathrm{P}<0.01$, *** $\mathrm{P}<0.001)$. Standard bars mean standard errors.

Figure 3 Upregulation of two NtJAZs and downregulation of nicotine pathway genes and $E R F 189$ in roots of $\mathrm{P}+\mathrm{T}$ transgenic seedlings

A-J, in roots of P+T-NL transgenic lines, the expression levels of NtJAZ1 (A) and $N t J A Z 3$ (B) were significantly upregulated; the expression level of $O D C 2$ (C) was not changed; while the expression levels of PMT1 (D), PMT2 (E) MPO (F), QPT (G), $A 622(\mathbf{H}), B B L s(\mathbf{I})$ (primer pairs designed for all three BBLs), and ERF189 (J) were significantly downregulated. K-T, in roots of $\mathrm{P}+\mathrm{T}-\mathrm{KY}$ transgenic lines, the expression levels of NtJAZ1 (K) and NtJAZ3 (L) were significantly upregulated, while, the expression levels $O D C 2(\mathbf{M}), P M T 1(\mathbf{N}), P M T 2(\mathbf{O}) M P O(\mathbf{P}), Q P T(\mathbf{Q}), A 622(\mathbf{R})$, $B B L s(\mathbf{S})$, and ERF189 (T) were significantly downregulated. Data of three transgenic lines are shown for both $\mathrm{P}+\mathrm{T}-\mathrm{NL}$ and $\mathrm{P}+\mathrm{T}-\mathrm{KY}$ genotypes. Wild type samples were pooled from five plants. Data of three lines are shown for vector control transgenic NL and KY171 plants. WT: wild type, P+T NL1, P+T NL2, P+T NL3: three lines of stacked PAP1 and TT8 transgenic NL plants, VC-1, 2, and 3-NL: three vector control transgenic NL lines, $\mathrm{P}+\mathrm{T}$ KY1, P+T KY2, P+T KY3: three lines of stacked PAP1 and 
1280

1281

1282

1283

1284

1285

1286

1287

1288

1289

1290

1291

1292

1293

1294

1295

1296

1297

1298

1299

1300

1301

1302

1303

TT8 transgenic KY171 plants. VC-1, 2, and 3-KY: three vector control transgenic KY171 lines.

Green and red bars represent wild type and vector control transgenic, and red P+T-NL and P+T-KY plants, respectively. Values represent the mean \pm S.D. $(n=3)$. Asterisks on top of the bars indicate that values are significantly lower or higher in red transgenic lines than in wild type plants $(* \mathrm{P}<0.05$, ** $\mathrm{P}<0.01, * * * \mathrm{P}<0.001)$. Standard bars mean standard errors.

Figure 4 Significant reduction of nicotine and nornicotine contents in leaves and roots of $\mathbf{P}+\mathbf{T}-\mathrm{NL}$ and $\mathbf{P}+\mathbf{T}-\mathrm{KY}$ seedlings. The contents of nicotine and nornicotine were significantly reduced in roots and leaves of both transgenic P+T-NL and P+TKY lines. A-C: reduction of nicotine (A), nornicotine $(\mathbf{B})$, and total nicotine and nornicotine (C) in leaves of three P+T-NL lines (1, 2, and 3); D-F: reduction of nicotine $(\mathbf{D})$, nornicotine $(\mathbf{E})$, and total nicotine and nornicotine $(\mathbf{F})$ in roots of three P+T-NL lines (1, 2, and 3); G-I: reduction of nicotine $(\mathbf{G})$, nornicotine $(\mathbf{H})$, and total nicotine and nornicotine (I) in leaves of three P+T-KY lines (1, 2 and 3); J-L: reduction of nicotine $(\mathbf{J})$, nornicotine $(\mathbf{K})$, and total nicotine and nornicotine $(\mathbf{L})$ in roots of three P+T-NL lines (1, 2, and 3). Bars labeled with "a" and "b" means pvalue less than 0.05 and bars labelled with the same lowcase "a" or "b" means no significant difference.

\section{Figure 5 Phenotypes of P+T-NL1 and P+T-KY1 plants versus their} corresponding wild-type tobacco plants in the field and of air-cured leaves. Field farming practice of four genotypes was performed in Oxford, North Carolina. A, 
1304

1305

1306

1307

1308

1309

1310

1311

1312

1313

1314

1315

1316

1317

1318

1319

1320

1321

1322

1323

1324

1325

1326

phenotypes of 30-day and 60-day old WT-NL vs. red P+T-NL1 plants. B, phenotypes of 30-day and 60-day old WT-KY171 vs. red P+T-KY1 plants. C-D, phenotypes of topped plants $(\mathbf{C})$ and leaves in air curing (D). Plant name abbreviations are WT-NL: wild-type Narrow Leaf Madole (NL) variety, P+T-NL1: Stacked PAP1 and TT8 transgenic NL line 1, WT-KY171: wild-type KY171 variety, and P+T-KY1: stacked $P A P 1$ and $T T 8$ transgenic KY line 1.

\section{Figure 6 Reduction of nicotine and four other alkaloids in cured leaves of red}

P+T-NL1 and P+T-KY1 tobacco plants. A-J, reduction of nicotine (A-B), nornicotine (C-D), anabasine (E-F), anatabine (G-H), and myosmine (I-J) in four leaf groups of P+T-NL (A, C, E, G, and I) and P+T-KY plants $(\mathbf{B}, \mathbf{D}, \mathbf{F}, \mathbf{H}$, and $\mathbf{J}) . \mathbf{K}-\mathrm{L}$, cartoons showing leaf groups harvested from both wild type $(\mathbf{K})$ and transgenic red plants (L).

Green and red bars represent wild type and red tobacco plants, respectively. B1, B2, $\mathrm{B} 3$, and B4 labels represent the $1^{\text {st }}, 2^{\text {nd }}, 3^{\text {rd }}$, and $4^{\text {th }}$ group of leaves from the base to the top of tobacco plant, respectively. Each metabolite in each leaf group was quantified from leaves harvested from 120 tobacco plants in three plots. One-way ANOVA test included in the SAS software was used to evaluate statistical significance. Bars labelled with lowcase "a" and " $b$ " mean significant difference between paired green and red bars $(n=120$, P-value less than 0.05). Percentages labeled in the top of red bars mean reduction compared to green bars. The standard bars mean standard deviation. Abbreviations, WT-NL: wild-type Narrow Leaf Madole (NL) variety, P+T-NL1: Stacked PAP1 and TT8 transgenic NL line 1, 
1327

1328

1329

1330

1331

1332

1333

WTKY: wild-type KY171 variety, and P+T-KY1: stacked PAP1 and TT8 transgenic KY line 1 .

Figure 7 Reduction of four individual and total TSNAs in leaves of red P+T-NL1 and P+T-KY1 tobacco plants. A-H, reduction of NNN (A-B), NNK (C-D), NAT (E-F), and NAB (G-H) in each leaf group of P+T-NL1 (A, C, E, and G) and P+TKY1 (B, D, F, and $\mathbf{H})$ plants; I-J, reduction of total TSNA contents summed from four individual TSNAs of each leaf group of P+T-NL1 (I) and P+T-KY1 (J) plants; K-L, reduction of total TSNA contents summed from four individual TSNAs of all leaves of P+T-NL1 (K) and P+T-KY1 (L) plants.

Green and red bars represent wild type and red tobacco plants, respectively. B1, B2, B3, and B4 labels represent the $1^{\text {st }}, 2^{\text {nd }}, 3^{\text {rd }}$, and $4^{\text {th }}$ group of leaves from the base to the top of plants, respectively. Each metabolite in each group of leaves was quantified from 120 tobacco plants. One-way ANOVA test included in the SAS software was used to evaluate statistical significance. Paired green and red bars labelled with lowcase "a" and "b" mean significant difference (n=120, P-value less than 0.05). Percentages labeled on the top of red bars indicate reduction levels compared to green bars. Abbreviation, WT-NL: Narrow Leaf Madole, WT-KY171: wild type KY171, P+T-NL: stacked PAP1 and TT8 transgenic NL line 1, and P+T-KY1: stacked PAP1 and TT8 transgenic KY171 line 1. 


\section{1 plants}

1352 Regulation pathways created in red tobacco plants are featured by red colored solid arrows, "X" shape, and "T" blocks. The stacked PAP1 and TT8 as well as the endogenous NtTTG1 encode MYB, bHLH, and WD40 proteins, respectively, which form a complex to regulate two biosynthetic pathways. The regulation pathway

1356 (function I) is the activation of biosynthesis of anthocyanin. The regulation pathway

1357 (function II) is the DPCR that the MYB-bHLH-WD40 complex upregulates the endogenous NtJAZs leading to the reduction of MYC2 and the downregulation of

$1359 E A R$ and the nicotine biosynthesis. In addition, The JA-JAZ-MYC regulatory pathway

1360 is indicated with green arrows starting with JA (greenish arrows). The feedback

1361 regulation of NtMYC2 indicated by a brownish bending arrow is proposed to activate 1362 the expression of NtJAZs. SCF: Skp/Cullin/F-box and COI1: coronatine insensitive 1. 
Figures

A A simplified biosynthetic pathway of anthocyanins and proanthocyanidins
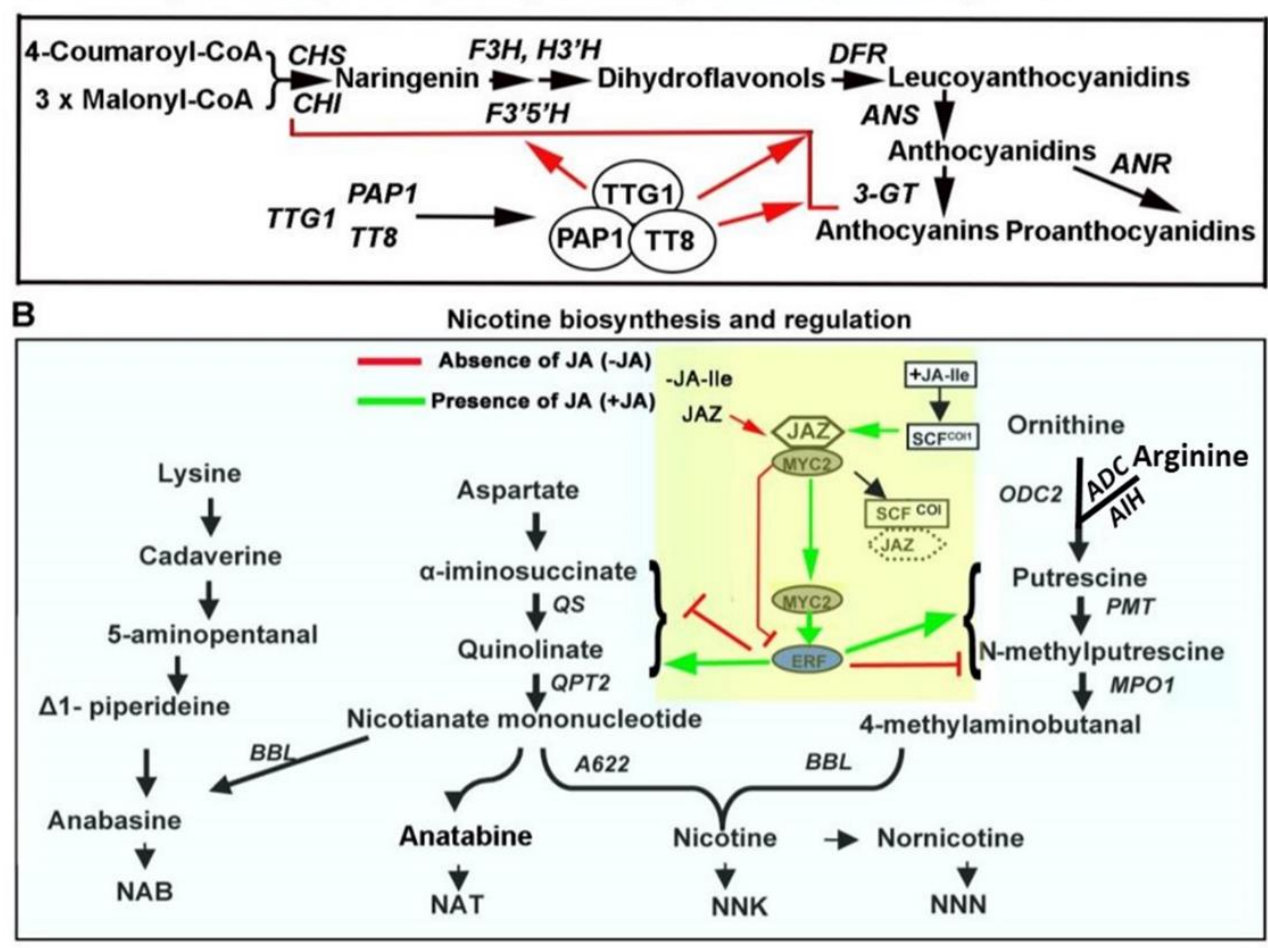

Figure 1 Biosynthetic pathways of nicotine, other tobacco alkaloids, and anthocyanin from the step catalyzed by CHS. PAP1, TT8, and TTG1 encode MYB75, bHLH, and WD40 to form a MYB-bHLH-WD40 complex that activates late pathway genes. Gene abbreviations are $C H S$ and $C H I$ : chalcone synthase and isomerase, $F 3 H$ : flavonone-3-hydroxylase, $F 3$ ' $H$ and $F 3$ '5 ' $H$ : flavonoid-3'-hydroxylase and

1373 3'5'hydroxylase, DFR: dihydroflavonol reductase, ANS: anthocyanidin synthase, 3-

$1374 G T$ : glycosyltransferase. ANR encodes anthocyanidin reductase, a key enzyme toward 1375 proanthocyanidin biosynthesis. Red lines and arrows highlight that the complex upregulates those steps. 
1377 B, the biosynthetic pathway of nicotine, nornicotine, and tobacco specific

1378 nitrosamines (TSNAs) and the regulation mechanism. MYC2 and EAR are two

1379 positive transcription factors that activate the expression of most pathway genes. In

1380 the absence of isoleucine-jasmonate (Ile-JA), NtJAZ1 and NtJAZ3 repressors bind to

1381 MYC2 to inhibit the activation of the pathway genes (red lines). In the presence of

1382 bioactive Ile-JA (green arrows), $\mathrm{SCF}^{\mathrm{COI} 1}$ perceives this plant hormone to form a new

1383 JA-SCF ${ }^{\mathrm{CO} 1}$ complex to bind to NtJAZs and lead NtJAZs to proteasomal

1384 ubiquitination. The degradation of NtJAZs releases MYC2 to activate pathway gene

1385 expression toward the biosynthesis of nicotine. Gene abbreviations are $A O$ : aspartate

1386 oxidase, $Q S$ : quinolinate synthase, $Q P T 2$ : quinolinate phosphoribosyltransferase 2,

$1387 A D C$ : arginine decarboxylase, $A I H$ : agmatine iminohydrolase, $O D C$ : ornithine

1388 decarboxylase, MPT1: N-methylputricine transferase 1, MPO1: methylputricine

1389 oxidase 1, A622: an isoflavone reductase like enzyme, $B B L$ : berberine bridge enzyme-

1390 like protein, and NND: nicotine $\mathrm{N}$-demethylase. 


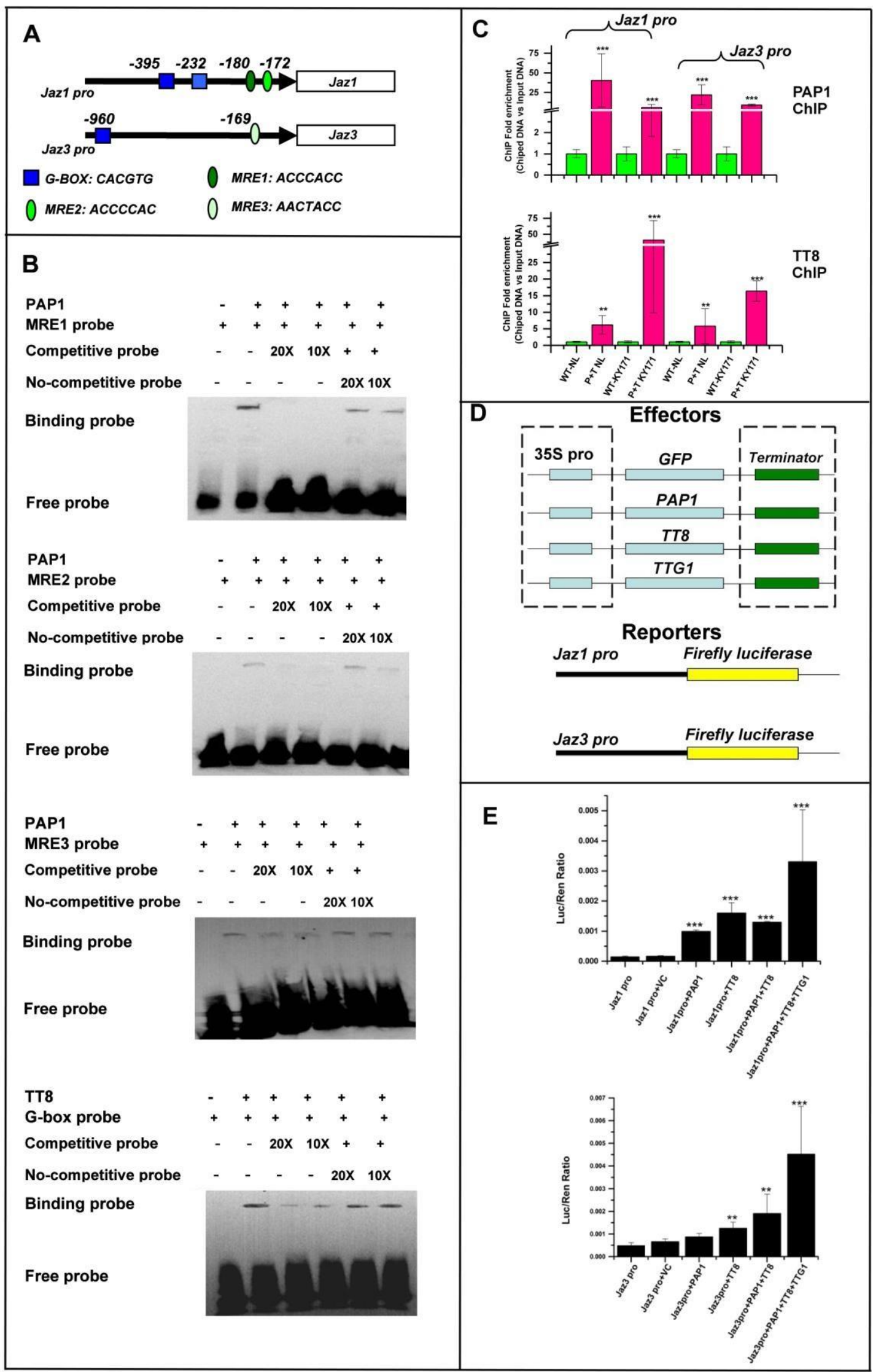


Figure 2 Binding and activation of NtJAZ1 and NtJAZ3 promoters by PAP1 and TT8 alone, PAP1 and TT8 together, and PAP1-TT8-WD40 complex. A, three MYB recognizing element (MRE) types and G-box were identified in the NtJAZ1 and NtJAZ3 promoters; B, electrophoretic mobility shift assay (EMSA) showed that PAP1 and TT8 bound to three MRE types and G-box of the NtJAZ1 and NtJAZ3 promoters, respectively. Competitive and non-competitive probes were used for binding of both TFs. $20 \times$ and 10x: concentrations of tested competitive and non-competitive probes 20 and 10 times as those MRE and G-box probes. C, fold change values from Chip-qPCR showed that both PAP1 and TT8 bound to the NtJAZ1 and NtJAZ3 promoters in vivo. Approximately 150 bp NtJAZ1 and NtJAZ3 promoter fragments containing both MRE and G-box were enriched with anti-HA antibodies in qRT-PCR analysis. The regions of NtJAZ1 and NtJAZ3 promoters that do not contain a MRE and G-box were used as negative controls. Green and red bars represent wild type and red tobacco plants, respectively. D, schematic diagrams shows four effector constructs (PK2GW7-GFP, PAP1, TT8 and TTG1) and two reporter constructs (pGreenII-0800-Jaz1pro and Jaz3pro) used for dual-luciferase assay. E, luciferase (LUC)/renilla (REN) luminescence ratios from dual luciferase assays showed that PAP1 and TT8 alone bound to and activated the promoters of both NtJAZ1 and NtJAZ3, two TF together increased promoter activity, and two TFs and WD40 together increased the highest activity. The promoters were fused to a firefly luciferase (reporter). The promoters of NtJAZ1 and NtJAZ3 genes were used in dual luciferase assay. Values represent the mean \pm S.D. $(n=5)$. Asterisks on top of bars indicate that the values are significantly higher than those of controls $(* \mathrm{P}<0.05, * * \mathrm{P}<0.01, * * * \mathrm{P}<0.001)$. Standard bars mean standard errors. 


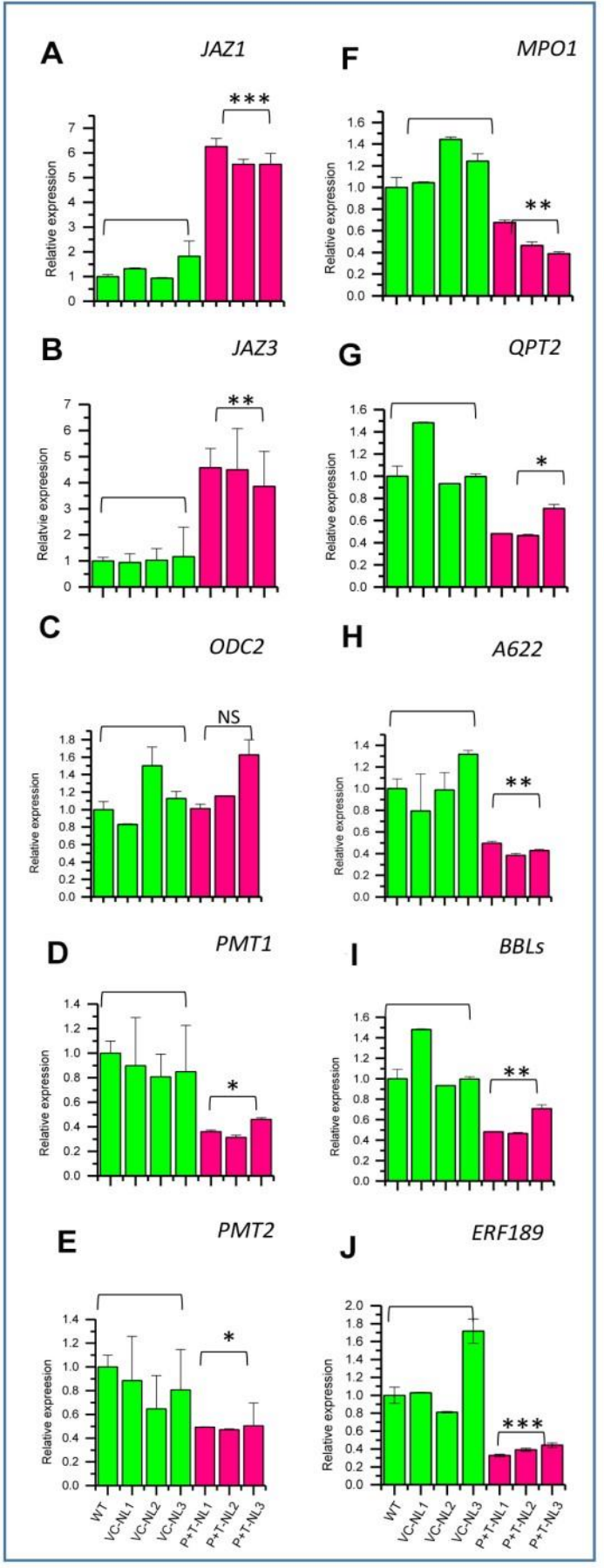

NL

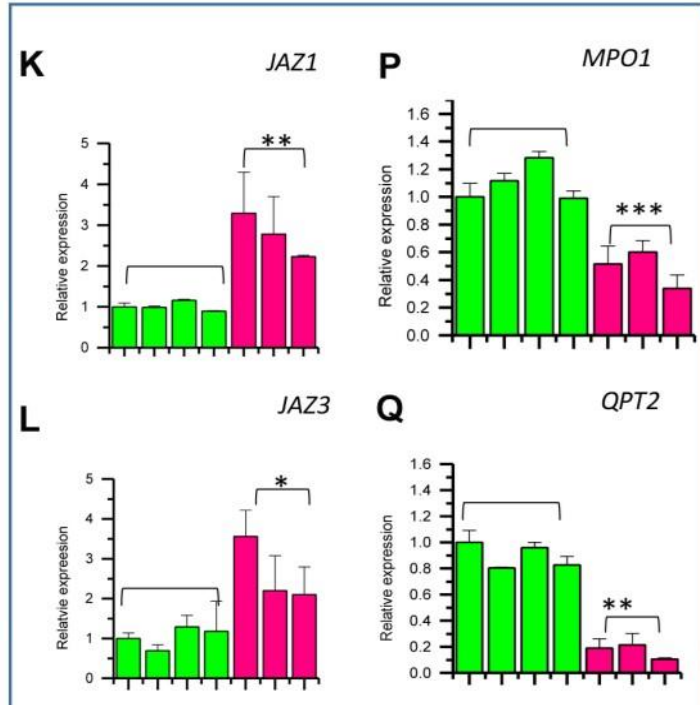

$\mathbf{M} \quad O D C 2 \quad \mathbf{R}$
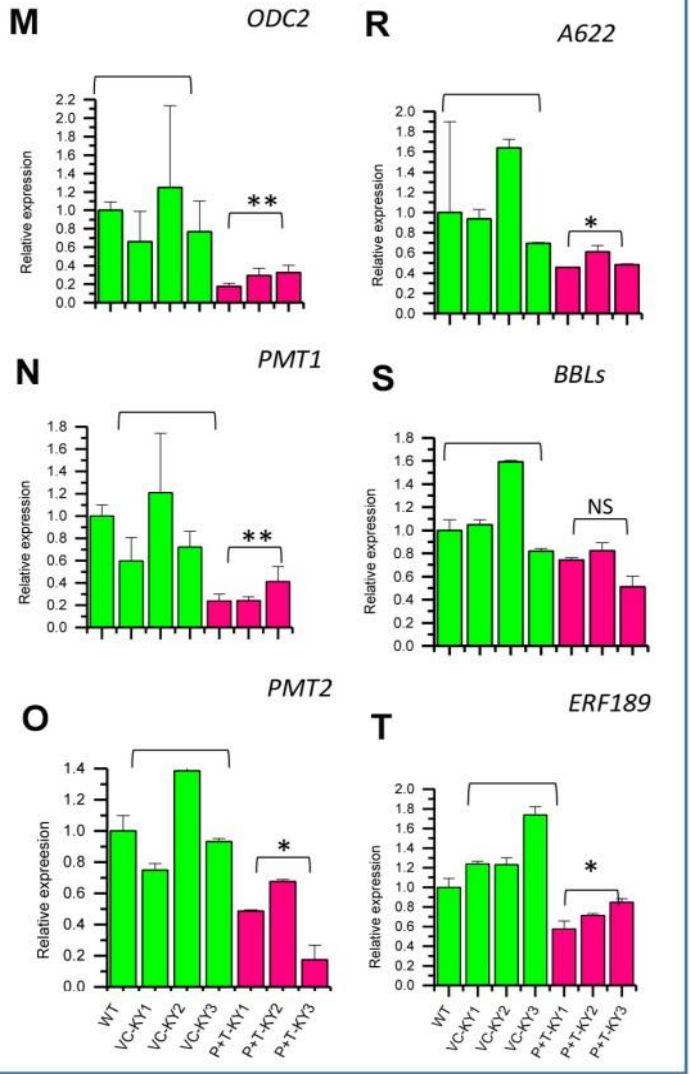

KY171

Figure 3 Upregulation of two NtJAZs and downregulation of six or seven nicotine pathway genes and ERF189 in roots of P+T transgenic seedlings shown by $\mathrm{qRT}-\mathrm{PCR}$

A-J, in roots of P+T-NL transgenic lines, the expression levels of NtJAZ1 (A) and NtJAZ3

(B) were significantly upregulated; the expression level of $O D C 2$ (C) was not changed; and 
the expression levels of PMT1 (D), PMT2 (E) MPO (F), QPT (G), A622 (H), BBLs (I) (primer pairs designed for all three BBLs), and $\operatorname{ERF} 189$ (J) were significantly downregulated. K-T, in roots of $\mathrm{P}+\mathrm{T}-\mathrm{KY}$ transgenic lines, the expression levels of NtJAZ1 (K) and NtJAZ3 (1) were significantly upregulated, and the expression levels $O D C 2(\mathbf{M})$, PMT1 (N), PMT2 (O) MPO (P), QPT (Q), A622 (R), BBLs $(\mathbf{S})$, and ERF189 (T) were significantly downregulated. Data of three transgenic lines are shown for both P+T-NL and P+T-KY genotypes. Wild type samples were pooled from five plants. Data of three lines are shown for vector control transgenic NL and KY171 plants. WT: wild type, P+T NL1, P+T NL2, P+T NL3: three lines of stacked PAP1 and TT8 transgenic NL plants, VC-1, 2, and 3NL: three vector control transgenic NL lines, P+T KY1, P+T KY2, P+T KY3: three lines of stacked PAP1 and TT8 transgenic KY171 plants. VC-1, 2, and 3-KY: three vector control transgenic KY171 lines.

Green and red bars represent wild type and vector control transgenic, and red P+T-NL and P+T-KY plants, respectively. Values represent the mean \pm S.D. $(n=3)$. Asterisks on top of the bars indicate that values are significantly lower or higher in red transgenic lines than in wild type plants $(* \mathrm{P}<0.05, * * \mathrm{P}<0.01, * * * \mathrm{P}<0.001)$. Standard bars mean standard errors. 
A

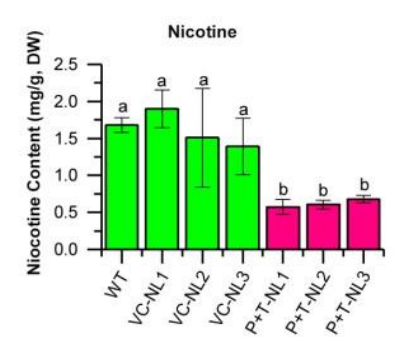

D

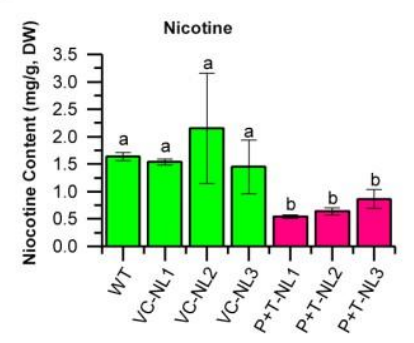

G

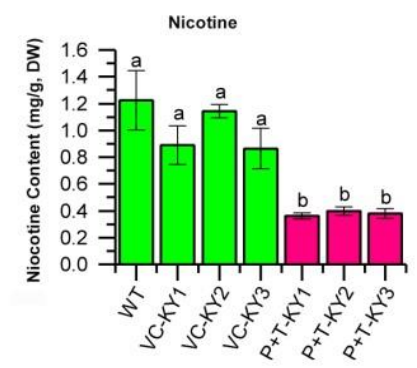

$\mathbf{J}$

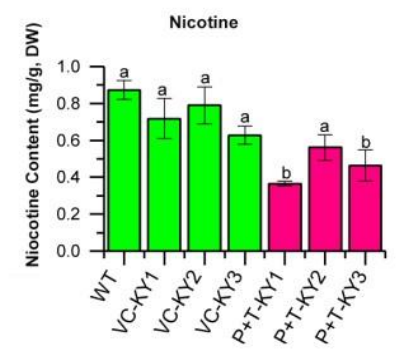

B

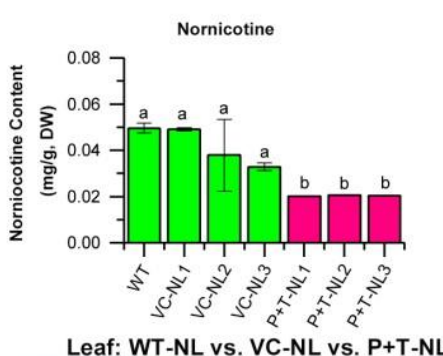

E

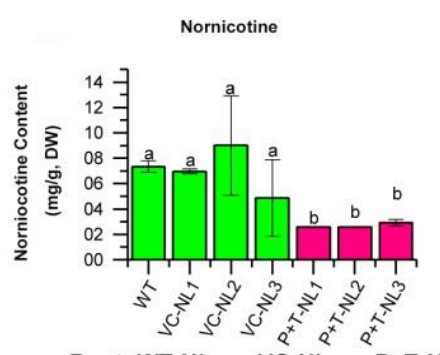

Root: WT-NL vs. VC-NL vs. P+T-NL

H

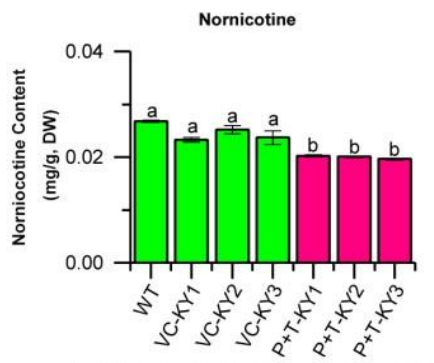

Leaf: WT-NL vs. VC-NL vs. P+T-KY
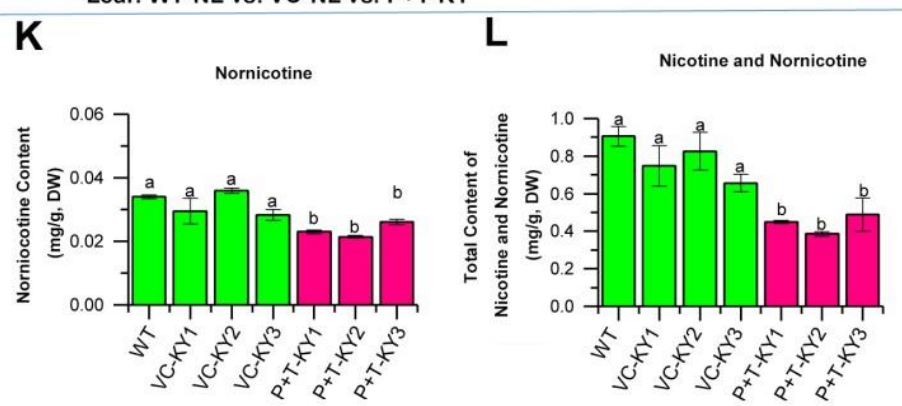

Root: WT-NL vs. VC-NL vs. P+T-KY

C

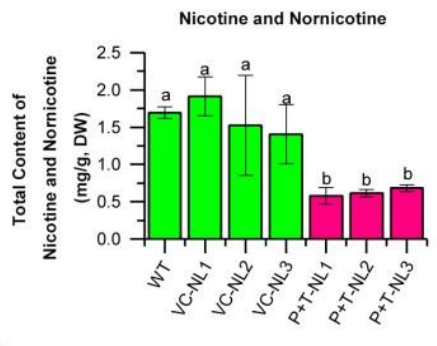

$\mathbf{F}$

Nicotine and Nornicotine

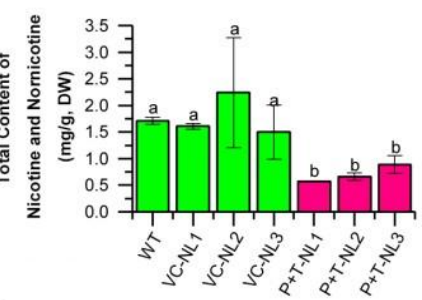

\section{I}

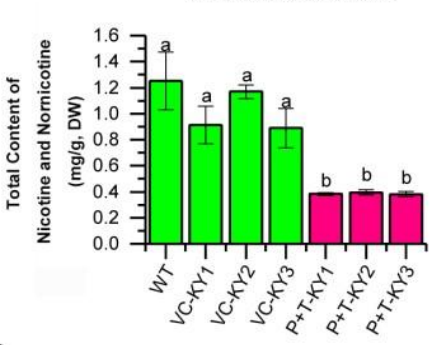

Figure 4 Significant reduction of nicotine and nornicotine contents in leaves and roots of

P+T-NL and P+T-KY seedlings. The contents of nicotine and nornicotine were significantly reduced in roots and leaves of both transgenic $\mathrm{P}+\mathrm{T}-\mathrm{NL}$ and $\mathrm{P}+\mathrm{T}-\mathrm{KY}$ lines. A-C: reduction of nicotine (A), nornicotine (B), and total nicotine and nornicotine $(\mathbf{C})$ in leaves of three P+TNL lines (1, 2, and 3); D-F: reduction of nicotine (D), nornicotine (E), and total nicotine and nornicotine (F) in roots of three P+T-NL lines (1, 2, and 3); G-I: reduction of nicotine (G), 
nornicotine $(\mathbf{H})$, and total nicotine and nornicotine $(\mathbf{I})$ in leaves of three P+T-KY lines $(1,2$

and 3); J-L: reduction of nicotine $(\mathbf{J})$, nornicotine $(\mathbf{K})$, and total nicotine and nornicotine $(\mathbf{L})$

in roots of three P+T-NL lines (1, 2, and 3). Bars labeled with "a" and "b" means p1314

value less than 0.05 and bars labelled with the same lowcase "a" or "b" means no 1315

significant difference.

1316 
A
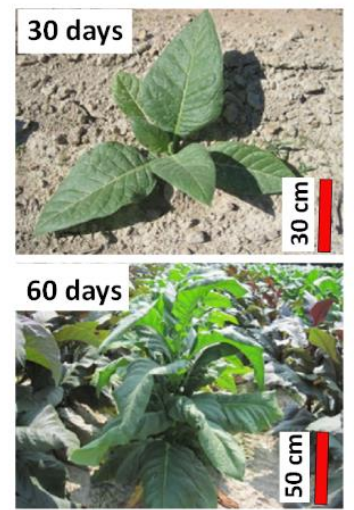

WT-NL

C

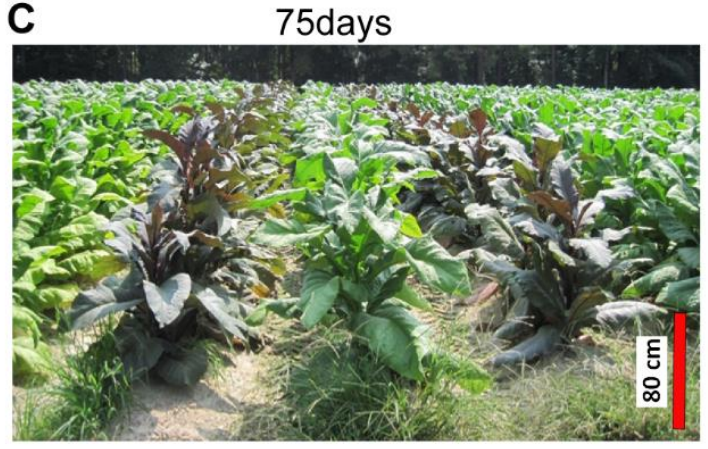

WT-NL P+T-NL1

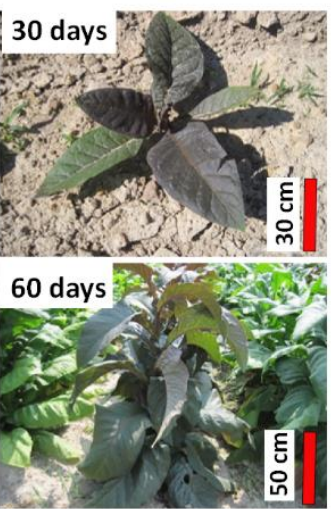

P+T-NL1

\section{B}
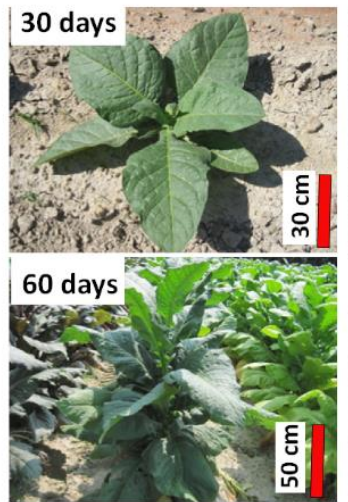

WT-KY171

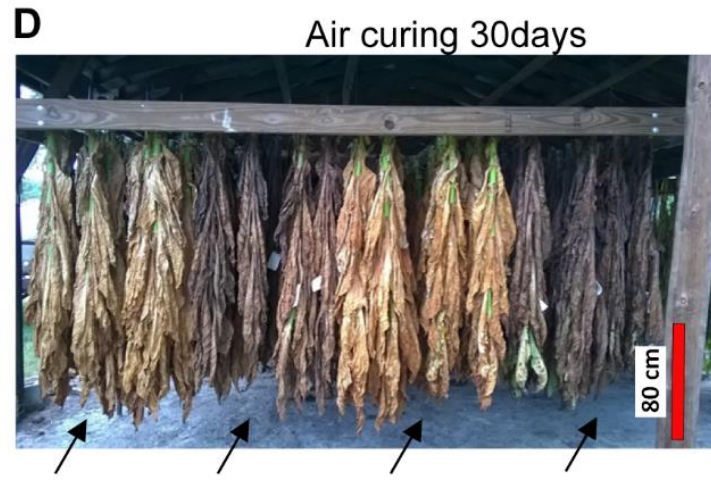

$\begin{array}{llll}\text { WT-NL } & \text { P+T-NL1 } & \text { WT-KY171 } & \text { P+T-KY1 }\end{array}$

farming practice of four genotypes was performed in Oxford, North Carolina. A,

1321 phenotypes of 30-day and 60-day old WT-NL vs. red P+T-NL1 plants. B, phenotypes

1322 of 30-day and 60-day old WT-KY171 vs. red P+T-KY1 plants. C-D, phenotypes of

1323 topped plants $(\mathbf{C})$ and leaves in air curing (D). Plant name abbreviations are WT-NL:

1324 wild-type Narrow Leaf Madole (NL) variety, P+T-NL1: Stacked PAP1 and TT8

1325 transgenic NL line 1, WT-KY171: wild-type KY171 variety, and P+T-KY1: stacked 
A

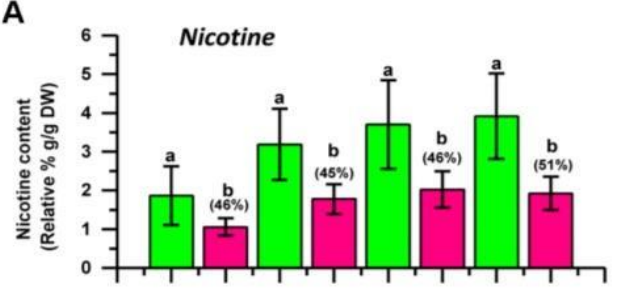

C

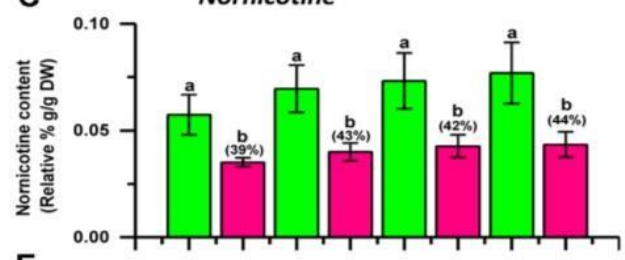

E

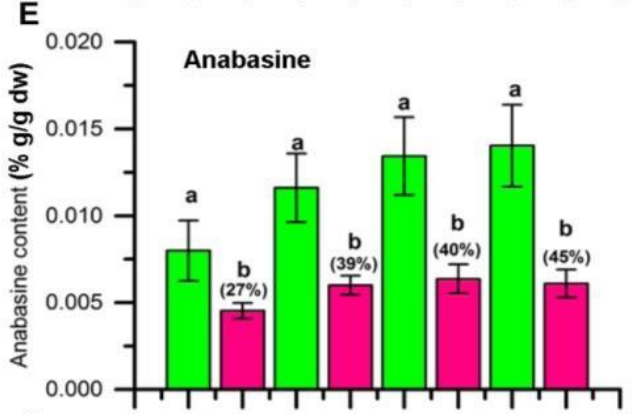

G

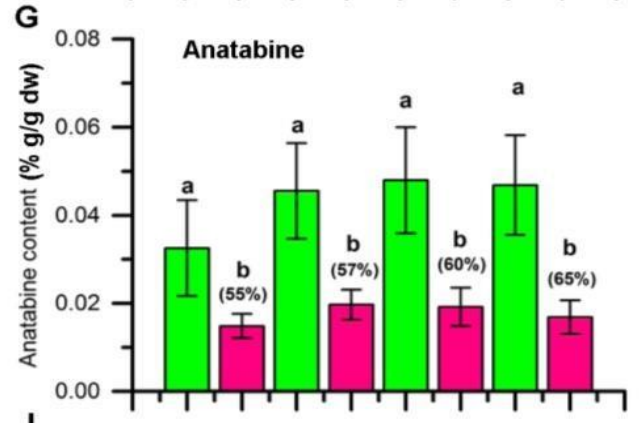

I 0.0067 Myosmine

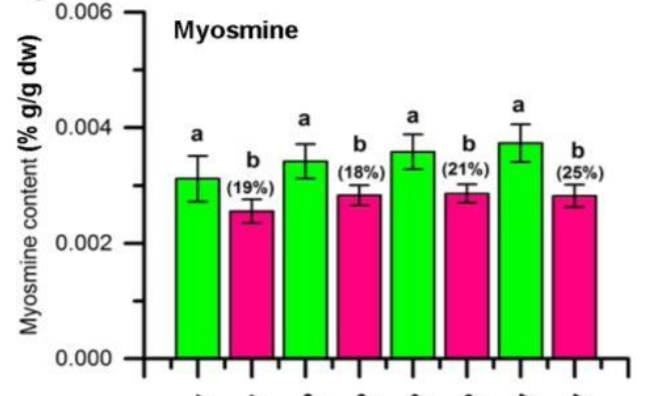

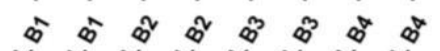

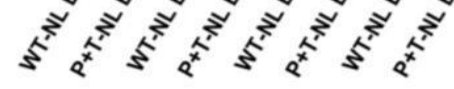

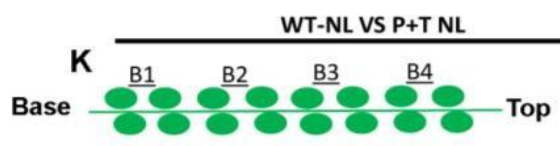

B
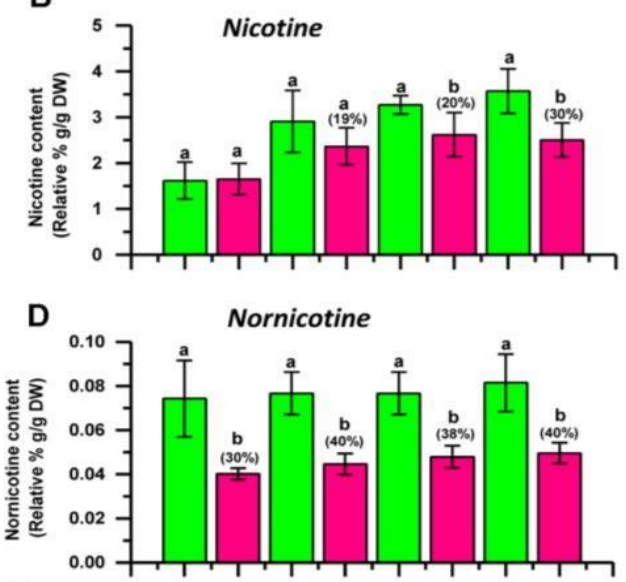

F 0.0207 Anabasine
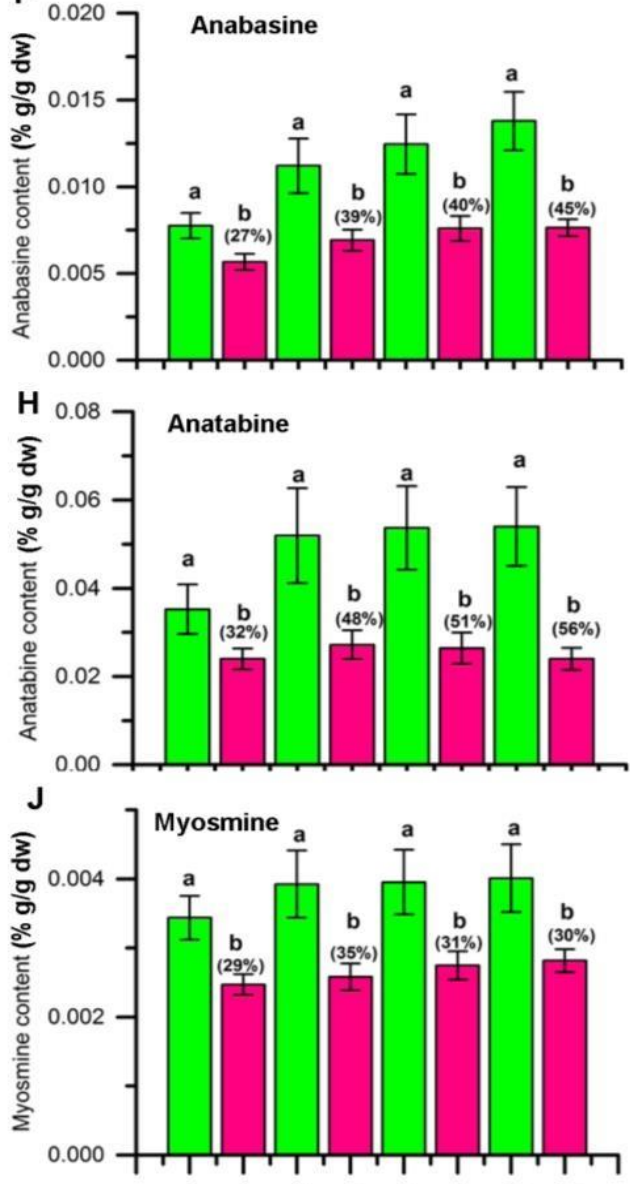

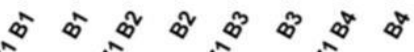

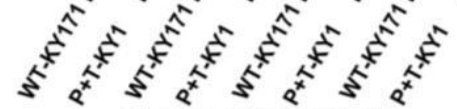

WT-KY171 VS P+T KY171

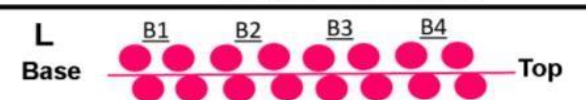


nornicotine (C-D), anabasine (E-F), anatabine (G-H), and myosmine (I-J) in four leaf

1333 plants $(\mathbf{L})$.

1334 Green and red bars represent wild type and red tobacco plants, respectively. B1, B2,

1335 B3, and B4 labels represent the $1^{\text {st }}, 2^{\text {nd }}, 3^{\text {rd }}$, and $4^{\text {th }}$ group of leaves from the base to

1336 the top of tobacco plant, respectively. Each metabolite in each leaf group was

1337 quantified from leaves harvested from 120 tobacco plants in three plots. One-way

1338 ANOVA test included in the SAS software was used to evaluate statistical

1339 significance. Bars labelled with lowcase "a" and "b" mean significant difference

1340 between paired green and red bars $(n=120$, P-value less than 0.05). Percentages

1341 labeled in the top of red bars mean reduction compared to green bars. The standard

1342 bars mean standard deviation. Abbreviations, WT-NL: wild-type Narrow Leaf

1343 Madole (NL) variety, P+T-NL1: Stacked PAP1 and TT8 transgenic NL line 1,

1344 WTKY: wild-type KY171 variety, and P+T-KY1: stacked PAP1 and TT8 transgenic

1345 KY line 1. 


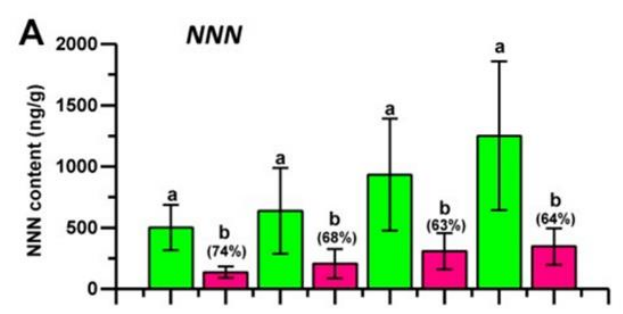

C

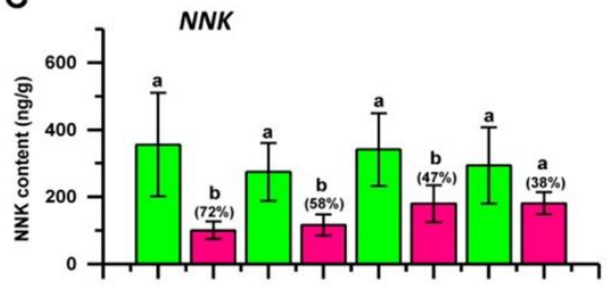

E

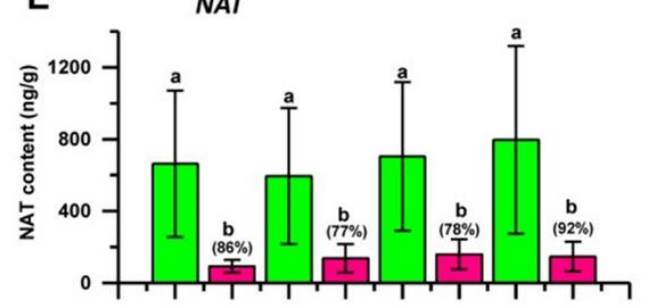

G

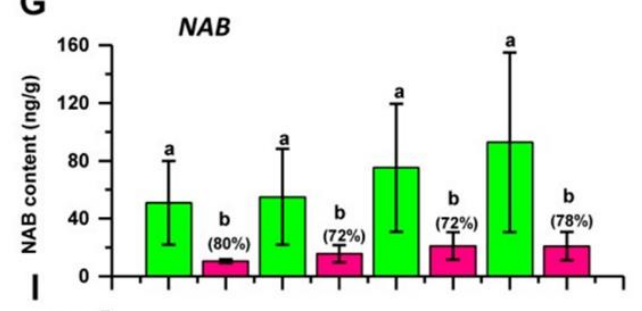

옹 2800 -

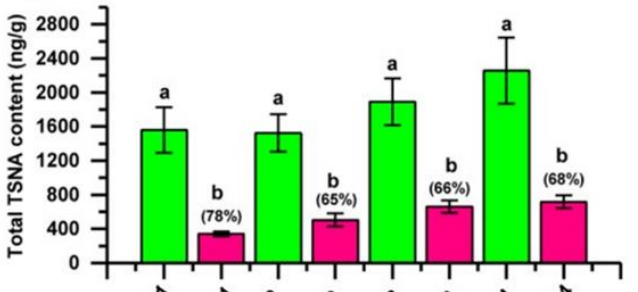

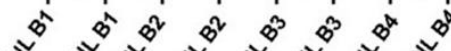

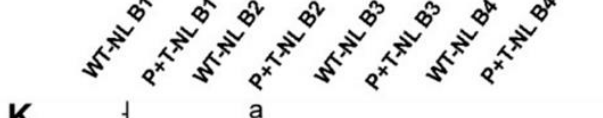

$$
\text { K }
$$

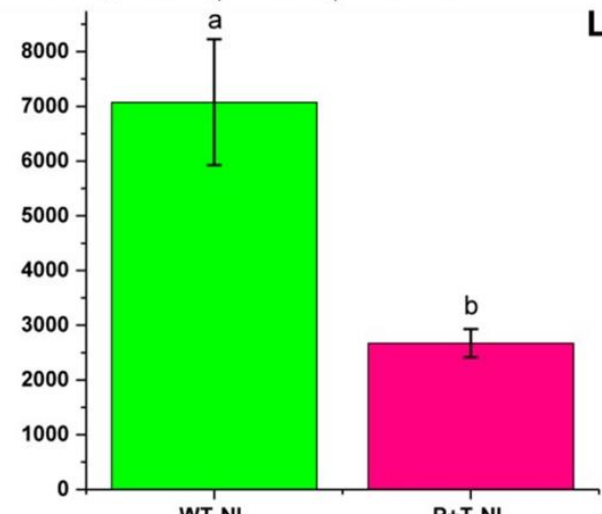

In All Leaves WT-NL VS P+T NL

L

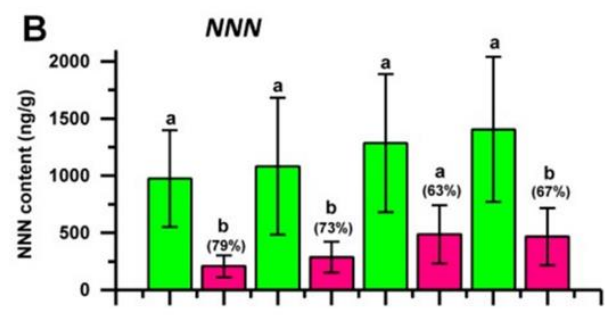

D NNK

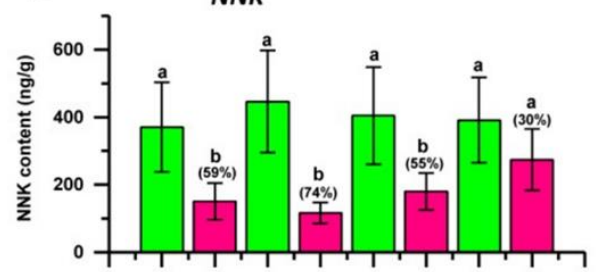

F NAT

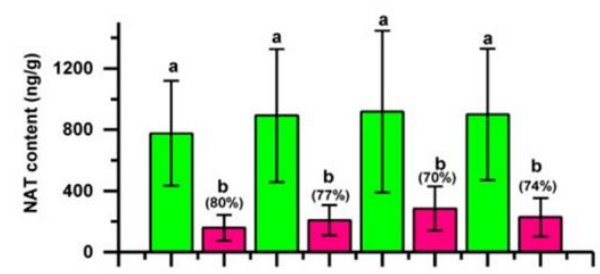

H
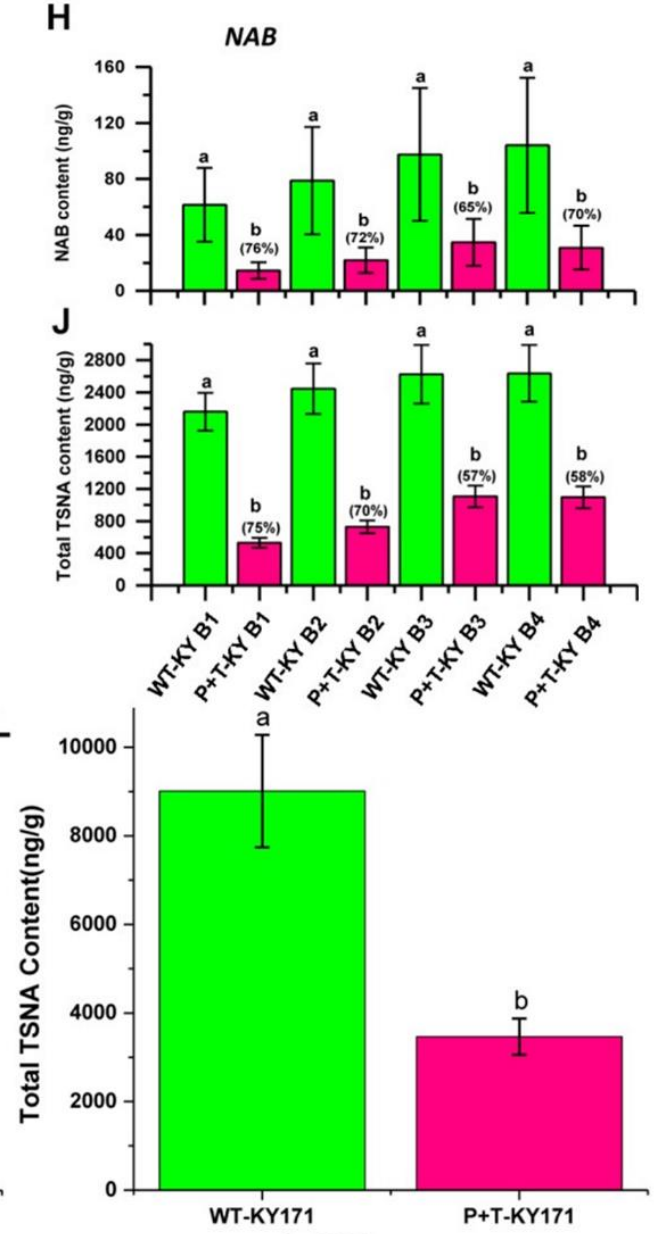

In All Leaves WT-KY171 VS P+T KY171 
Figure 7 Reduction of four individual and total TSNAs in leaves of red P+T-NL1 and

P+T-KY1 tobacco plants. A-H, reduction of NNN (A-B), NNK (C-D), NAT (E-F), and

NAB $(\mathbf{G}-\mathbf{H})$ in each leaf group of P+T-NL1 (A, C, E, and $\mathbf{G})$ and P+TKY1 $(\mathbf{B}, \mathbf{D}, \mathbf{F}$, and $\mathbf{H})$

1352 plants; I-J, reduction of total TSNA contents summed from four individual TSNAs of each

1353 leaf group of P+T-NL1 (I) and P+T-KY1 (J) plants; K-L, reduction of average total TSNA

1354 contents summed from four individual TSNAs of all leaves of P+T-NL1 (K) and P+T-KY1

1355

(L) plants.

1356 Green and red bars represent wild type and red tobacco plants, respectively. B1, B2, B3, and

1357 B4 labels represent the $1^{\text {st }}, 2^{\text {nd }}, 3^{\text {rd }}$, and $4^{\text {th }}$ group of leaves from the base to the top of plants, 1358 respectively. Each metabolite in each group of leaves was quantified from 120 tobacco

1359 plants. One-way ANOVA test included in the SAS software was used to evaluate statistical 1360 significance. Paired green and red bars labelled with lowcase "a" and " $b$ " mean significant 1361 difference ( $n=120, P$-value less than 0.05$)$. Percentages labeled on the top of red bars indicate 1362 reduction levels compared to green bars. Abbreviation, WT-NL: Narrow Leaf Madole, WT1363 KY171: wild type KY171, P+T-NL: stacked PAP1 and TT8 transgenic NL line 1, and P+T1364 KY1: stacked PAP1 and TT8 transgenic KY171 line 1. 


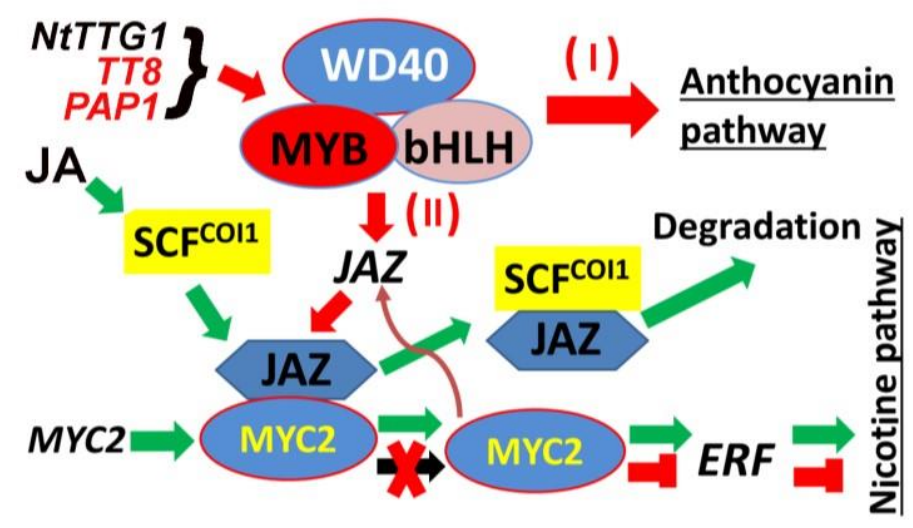

Figure 8 A model showing the distant pathway-cross regulation (DPCR) created for the

\section{downregulation of the biosynthesis of tobacco alkaloids in red tobacco plants}

Regulation pathways created in red tobacco plants are featured by red colored solid arrows, "X" shape, and "T" blocks. The stacked PAPl and TT8 as well as the endogenous NtTTG1 encode MYB, bHLH, and WD40 proteins, respectively, which form a complex to regulate two biosynthetic pathways. The regulation pathway (function I) is the activation of biosynthesis of anthocyanin. The regulation pathway (function II) is the DPCR that the MYB-bHLH-WD40 complex upregulates the endogenous NtJAZs leading to the reduction of MYC2 and the downregulation of EAR and the nicotine biosynthesis. In addition, The JAJAZ-MYC regulatory pathway is indicated with green arrows starting with JA (greenish arrows). The feedback regulation of NtMYC2 indicated by a brownish bending arrow is proposed to activate the expression of NtJAZs. SCF: Skp/Cullin/F-box and COI1: coronatine insensitive 1. 